\title{
Detection and Implications of Laser-Induced Raman Scattering at Astronomical Observatories
}

Frédéric P. A. Vogt, ${ }^{1, *}$ Domenico Bonaccini Calia, ${ }^{2}$ Wolfgang Hackenberg, ${ }^{2}$ Cyrielle Opitom, ${ }^{1}$ Mauro Comin, ${ }^{2}$ Linda Schmidtobreik, ${ }^{1}$ Jonathan Smoker, ${ }^{1}$ Israel Blanchard, ${ }^{1}$ Marcela Espinoza Contreras, ${ }^{1}$ Ivan Aranda, ${ }^{1}$ Julien Milli, ${ }^{1}$ Yara L. Jaffe, ${ }^{1}$ Fernando Selman, ${ }^{1}$ Johann Kolb, ${ }^{1}$ Pascale Hibon, ${ }^{1}$ Harald Kuntschner, ${ }^{2}$ and Pierre-Yves Madec ${ }^{2}$

${ }^{1}$ European Southern Observatory, Avenida Alonso de Córdova 3107, 7630355 Vitacura, Santiago, Chile

${ }^{2}$ European Southern Observatory, Karl-Schwarzschild-Straße 2, 85748 Garching, Germany

(Received 28 March 2017; published 22 June 2017)

Laser guide stars employed at astronomical observatories provide artificial wavefront reference sources to help correct (in part) the impact of atmospheric turbulence on astrophysical observations. Following the recent commissioning of the 4 Laser Guide Star Facility (4LGSF) on Unit Telescope 4 (UT4) of the Very Large Telescope (VLT), we characterize the spectral signature of the uplink beams from the $22-\mathrm{W}$ lasers to assess the impact of laser scattering from the 4LGSF on science observations. We use the Multi-Unit Spectroscopic Explorer (MUSE) optical integral field spectrograph mounted on the Nasmyth B focus of UT4 to acquire spectra at a resolution of $R \cong 3000$ of the uplink laser beams over the wavelength range of $4750 \AA-9350 \AA$. We report the first detection of laser-induced Raman scattering by $\mathrm{N}_{2}, \mathrm{O}_{2}, \mathrm{CO}_{2}, \mathrm{H}_{2} \mathrm{O}$, and (tentatively) $\mathrm{CH}_{4}$ molecules in the atmosphere above the astronomical observatory of Cerro Paranal. In particular, our observations reveal the characteristic spectral signature of laser photons-but $480 \AA$ to $2210 \AA$ redder than the original laser wavelength of $5889.959 \AA$-landing on the 8.2-m primary mirror of UT4 after being Raman-scattered on their way up to the sodium layer. Laser-induced Raman scattering, a phenomenon not usually discussed in the astronomical context, is not unique to the observatory of Cerro Paranal, but it is common to any astronomical telescope employing a laser guide star (LGS) system. It is thus essential for any optical spectrograph coupled to a LGS system to thoroughly handle the possibility of a Raman spectral contamination via a proper baffling of the instrument and suitable calibrations procedures. These considerations are particularly applicable for the HARMONI optical spectrograph on the upcoming Extremely Large Telescope (ELT). At sites hosting multiple telescopes, laser-collisionprediction tools should also account for the presence of Raman emission from the uplink laser beam(s) to avoid the unintentional contamination of observations acquired with telescopes in the vicinity of a LGS system.

DOI: 10.1103/PhysRevX.7.021044

\section{INTRODUCTION}

The past few years have seen the emergence of numerous laser guide star (LGS) systems at astronomical observatories [1,2]. These provide artificial wavefront reference sources to help alleviate the impact of atmospheric turbulence on astronomical observations [3,4]. A cleaner wavefront not only leads to an enhancement in image quality; it also improves the detection limit of the telescope by further concentrating the light of point sources, a crucial advantage for the detection (from the ground) of galaxies at the highest redshifts, for example. Most of the existing LGS

\section{*fvogt@eso.org}

Published by the American Physical Society under the terms of the Creative Commons Attribution 4.0 International license. Further distribution of this work must maintain attribution to the author(s) and the published article's title, journal citation, and DOI.
Subject Areas: Astrophysics,

Atomic and Molecular Physics,

Interdisciplinary Physics systems are available to the general community of observers and are thus used regularly in operations [5]. Such is the case at the W.M. Keck telescopes [6,7], the Gemini North and South telescopes [8-12], the Subaru telescope [13,14], and the Very Large Telescope (VLT) [15].

The 4 Laser Guide Star Facility (4LGSF) [16] consists of four laser guide star units (LGSUs), each comprised of a stand-alone 22-W laser [17-19], a launch telescope, steering mechanisms, and dedicated control electronics. These systems are an integral part of the Adaptive Optics Facility (AOF) [20] currently being installed on the Unit Telescope 4 (UT4) of the VLT on Cerro Paranal in Chile. In addition to the 4LGSF, the AOF also comprises a new deformable secondary mirror $[21,22]$ and the adaptive optics modules GALACSI [23,24] and GRAAL [25-27].

The AOF is intended to feed corrected wavefronts to the MUSE optical integral field spectrograph (spectral range: $4750 \AA-9350 \AA$ ) [28] and the HAWK-I near-IR wide-field imager [29,30]. Altogether, these systems allow us to correct, on the fly, the effect of atmospheric turbulence 
over several arc min using a square asterism of 4 LGSs, the spatial extent of which is adjustable to the respective field of views of MUSE [7.5 arc sec in narrow-field mode (NFM); 60 arc sec in wide-field mode (WFM)] and HAWK-I (7.5 arc min). Specifically, the adaptive optics corrections to be provided by the AOF are (a) laser tomography for the MUSE NFM and (b) ground-layer correction for the MUSE WFM and HAWK-I [31].

As the power of LGS systems increases, so does their potential for collateral damage on the associated astrophysical observations. In the case of Cerro Paranal, the $22 \mathrm{~W}$ of the 4LGSF lasers represent a factor of 4 increase in the on-sky power compared to the PARLA laser [15] used with the SINFONI infrared integral field spectrograph [32,33] on UT4 since March 2013. This power increase, the fact that the 4LGSF lasers will be used (at times) with an optical spectrograph, and the presence of numerous telescopes in the immediate vicinity of UT4 on Cerro Paranal motivated the spectral analysis of the 4LGSF uplink beams presented in this article. In particular, the present analysis distinguishes itself from previous observations targeting LGS systems [34-36] in that (1) it does not restrict itself to the immediate spectral vicinity of the laser wavelength, and (2) it focuses on the existence and consequences of inelastic Raman scattering physics (in addition to elastic Rayleigh and Mie scattering) associated with the use of LGS systems, a phenomena hitherto often overlooked within the astrophysics community.

This article is structured as follows. Our initial detection of laser-induced Raman scattered photons by $\mathrm{N}_{2}$ and $\mathrm{O}_{2}$ molecules above Cerro Paranal is described in Sec. II. We discuss our search for the signatures of Raman scattering from additional molecules in Sec. III and quantify the fluxes of the different Raman lines in Sec. IV. We conclude by discussing the impact of laser-induced Raman scattering physics on astrophysical observations in Sec. V. Unless mentioned otherwise, all wavelengths are quoted in air at a pressure of $1 \mathrm{~atm}$ and a temperature of $15^{\circ} \mathrm{C}$.

\section{FIRST DETECTION OF LASER-INDUCED ATMOSPHERIC RAMAN SCATTERING AT CERRO PARANAL}

We undertook dedicated observations combining the newly installed 4LGSF systems with the MUSE integral field spectrograph (located on the Nasmyth B focus of UT4) in June, November, and December of 2016, as part of the 4LGSF/AOF ongoing commissioning activities. These observations were designed to derive an initial characterization of the possible impact of laser scattering from the 4LGSF on science observations. We note that these observations were performed prior to the installation of the GALACSI AO module (which has since been inserted directly ahead of MUSE in the light path). All observations were reduced using biases, flat fields (including both lamp and sky flats), and arc lamp exposures acquired as part of the regular calibration plan of MUSE. The data were fluxcalibrated using dedicated observations of flux standard stars (one per night) taken from the MUSE list of standard stars.

At the laser wavelength, the laser uplink beams are visible through elastic, nonisotropic, polarization-preserving Rayleigh scattering of the laser photons by the air molecules, and through nonisotropic, higher-intensity, Mie scattering by dust particles. In the specific case of the 4LGSF, $18 \mathrm{~W}$ are emitted by each LGSU at the laser main carrier wavelength $\lambda_{4 \mathrm{LGSF}, \text { vacuum }}=5891.5912 \AA$ to excite the $\mathrm{D}_{2}$ a transition of sodium atoms. An additional $4 \mathrm{~W}$ are emitted in two lines located at $1.713 \mathrm{GHz}$ on either side of the main line at $5891.5714 \AA$ and $5891.6110 \AA$ (with $2 \mathrm{~W}$ in each line). Emitting $10 \%$ of the laser power at $5891.5714 \AA$ is specifically designed to counteract the depopulation of the sodium atoms from the ${ }^{2} S_{1 / 2} \mathrm{~F}=2$ to $\mathrm{F}=1$ ground state by repumping them via the $\mathrm{D}_{2} \mathrm{~b}$ transition (where $\mathrm{F}$ is the total atomic angular momentum quantum number; see Ref. [37]). These three lines are essentially unresolved at the spectral resolution of MUSE, so in the remainder of this article, we simply refer to the 4LGSF laser carrier wavelength in air, $\lambda_{4 \mathrm{LGSF}}=5889.959 \AA$, where

$$
\lambda_{4 \mathrm{LGSF}}=\frac{\lambda_{4 \mathrm{LGSF}, \mathrm{vacuum}}}{n},
$$

with $n$ the refractive index of dry air at a pressure of $1 \mathrm{~atm}$ and a temperature of $15^{\circ} \mathrm{C}$ defined by [38]

$n=1+\frac{8.34254}{10^{5}}+\frac{2.406147}{10^{2} \times\left(130-s^{2}\right)}+\frac{1.5998}{10^{4} \times\left(38.9-s^{2}\right)}$,

with

$$
s=\frac{10^{4}}{\lambda_{4 \mathrm{LGSF}, \text { vacuum }}} .
$$

In normal operations, MUSE will employ dedicated, nearly $225 \AA$-wide, notch filters - centered on the LGS frequency - to block $99.7 \pm 0.2 \%$ of the light emitted by the uplink laser beams. Comparing two back-to-back 60-sec MUSE exposures (acquired with and without the 4LGSF lasers propagating) readily reveals the presence of a residual narrow and unresolved emission line at $\lambda_{4 \mathrm{LGSF}}$ (see Fig. 1). The presence of the notch filter in the light path (leading to the nearly $225-\AA$ gap in the spectrum) reduces the intensity of this laser line, without fully blocking it. As such, this line effectively offers an excellent wavelength and spectral resolution reference, given that the laser wavelength is controlled with a root-mean-square error of $0.03 \mathrm{~m} \AA$.

We also detect two additional spectral features associated with the use of the $4 \mathrm{LGSF}$ at $\lambda \cong 6484 \AA$ and $\lambda \cong 6827 \AA$. 


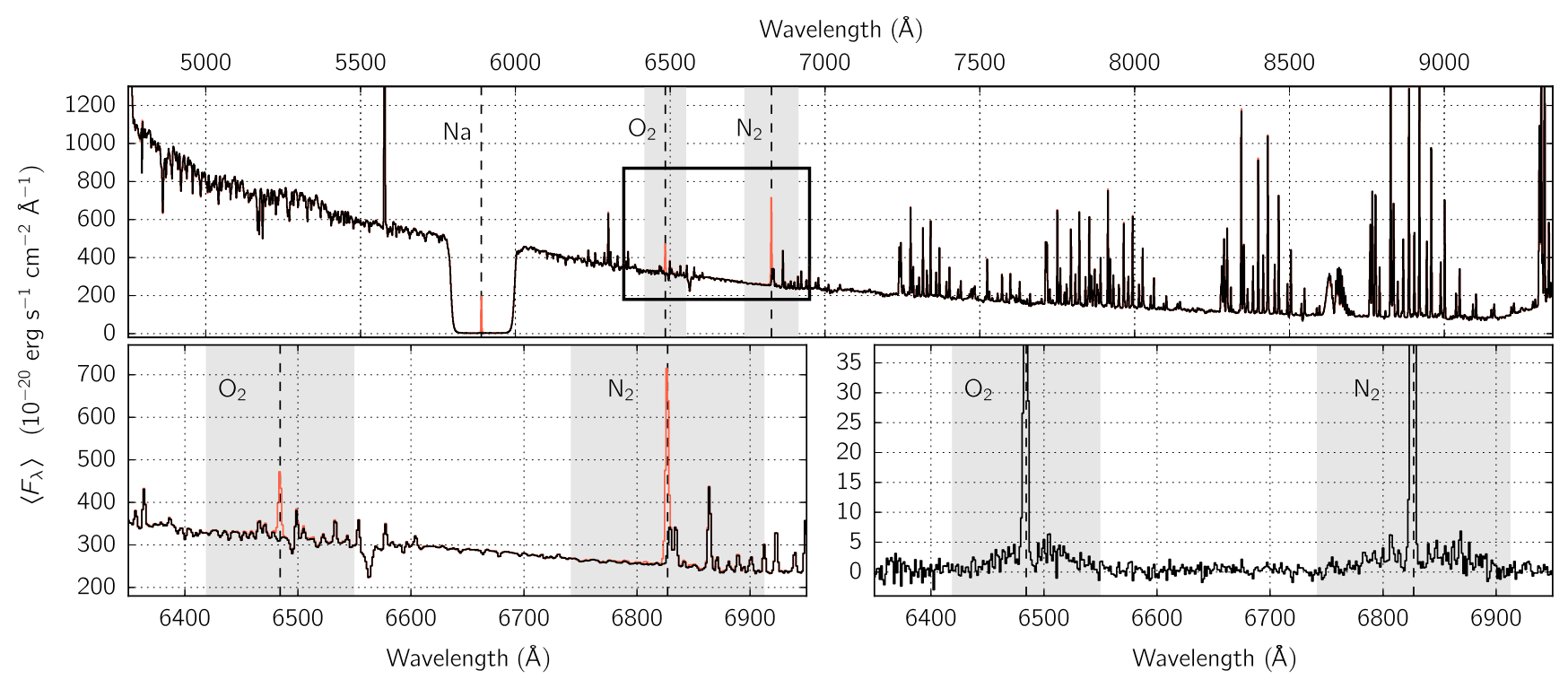

FIG. 1. Top panel: Mean MUSE spectra [in the WFM-nominal (WFM-N) setup] constructed from a 60-s exposure on an empty sky field at an air mass of around 1.8, with and without the 4LGSF lasers propagating (red and black curves, respectively), under full-moon conditions. In addition to the residual laser line (visible through Rayleigh and Mie scattering in the laser beams) at $\lambda_{4 \mathrm{LGSF}}$ (labeled "Na"), spectral features resulting from the inelastic Raman scattering of laser photons by the $\mathrm{O}_{2}$ and $\mathrm{N}_{2}$ photons are present at $6484.39 \AA$ and $6827.17 \AA$ (respectively) when the 4LGSF lasers are propagating. Bottom left panel: Same as top panel, but zooming in on the $6350 \AA$ $6950 \AA$ spectral range. Bottom right panel: Difference between the spectra of the top panel, revealing the rotational wings (i.e., the $S$ branch and the $O$ branch) surrounding the central vibrational peak (i.e., the $Q$ branch) of the Raman lines for both the $\mathrm{O}_{2}$ and $\mathrm{N}_{2}$ molecules. The spectral extent of the Raman contamination (including both the vibrational central peak and rotational sidebands) is traced using greyed-out areas in all panels. The box in the top panel shows the extent of the bottom panels.

These features consist of (1) a sharp and unresolved emission line with a peak flux density similar to that of the brightest sky emission lines in the $7000 \AA-8000 \AA$ spectral range, and (2) broad wings at a level of $\lesssim 5 \times$ $10^{-20} \mathrm{erg} \mathrm{s}^{-1} \mathrm{~cm}^{-2} \AA^{-1}$ spanning about $130 \AA$ and $190 \AA$, respectively. These spectral features evidently result from the inelastic Raman scattering by the $\mathrm{O}_{2}$ and $\mathrm{N}_{2}$ molecules of the laser photons while on their way up to the sodium layer: a process seen easily at high peak powers with, e.g., pulsed light detection and ranging (LIDAR) lasers, and exploited for many years in atmospheric physics experiments (see, e.g., Refs. [39-45]). Specifically, through the inelastic collision with $\mathrm{O}_{2}$ or $\mathrm{N}_{2}$ molecules, laser photons lose a quantized amount of energy through the excitation of the fundamental $\nu_{1 \leftarrow 0}$ rotational-vibrational (rovibrational) modes of these diatomic molecules [46-48]. The nature of Raman scattering is such that the molecule responsible for a specific spectral line is not tied to the absolute wavelength of the line but instead to its shift from the exciting (laser) wavelength. Indeed, the spectral lines in our MUSE observations are in perfect agreement with the tabulated Raman shifts of $\Delta \nu_{0, \mathrm{O}_{2}}=1556.4 \mathrm{~cm}^{-1}$ and $\Delta \nu_{0, \mathrm{~N}_{2}}=2330.7 \mathrm{~cm}^{-1}$ for $\mathrm{O}_{2}$ and $\mathrm{N}_{2}$ [47], implying resulting (post-collision) photon wavelengths of $6484.39 \AA$ and $6827.17 \AA$.

In normal operations, the four LGSs are located outside of the MUSE field of view (about 62 arc sec from the center in WFM; see Fig. 2). As such, the Rayleigh and Raman signals in the MUSE WFM observations presented in Fig. 1 (i.e., with the LGSs outside of the instrument field of view) are best described as a residual glow throughout the field of view. As illustrated in Fig. 3, we note that (1) the intensity of the Rayleigh (and Raman) glow is strongly dependent on the size of the 4LGSF asterism (probed out to its physical limit of about 440 arc sec in radius set by the optomechanical range of the 4LGSU field steering devices), and (2) there is no evidence for the Rayleigh (and Raman) glow to be dependent on the altitude of the observation. Preliminary follow-up observations performed on April 15, 2017 during the second commissioning run of GALACSI reveal that the installation of this $\mathrm{AO}$ module does not have any noticeable influence on the intensity of the Raman lines seen by MUSE. The commissioning of GALACSI and the AO mode of MUSE is ongoing; a detailed and comprehensive characterization of the intensity and impact of Raman lines on MUSE AO observations (both in the WFM and NFM) is thus clearly not feasible just yet. This aspect will be thoroughly investigated during commissioning observations and reported on separately.

The absence of GALACSI in the light path during the 4LGSF commissioning runs of June, November, and December 2016 did not, however, preclude a detailed characterization of the Raman emission lines themselves. For test purposes, we acquired a 60 -sec MUSE exposure with one LGS guide star voluntarily placed within the 


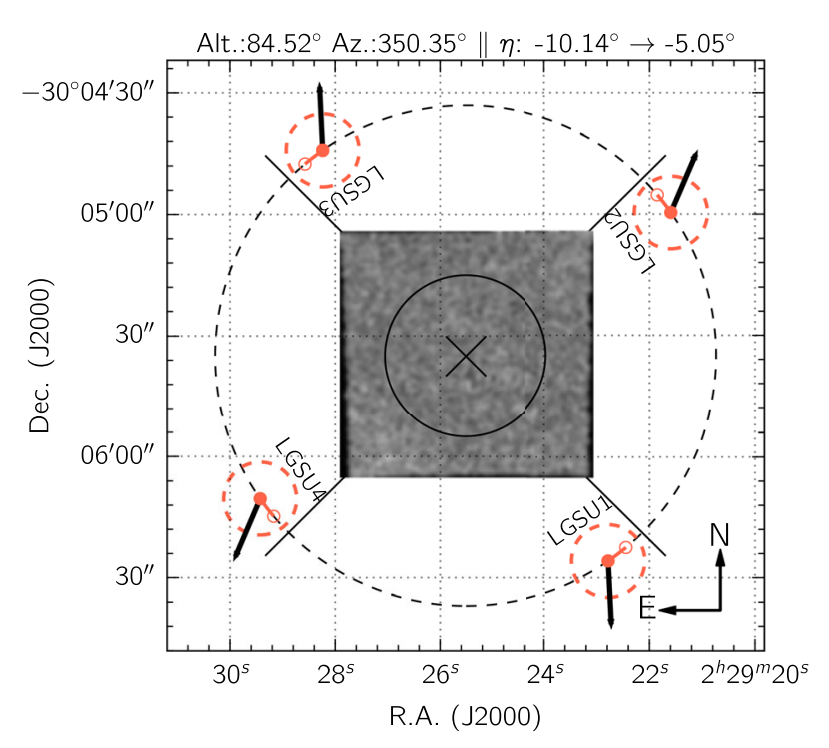

FIG. 2. Location of the LGSs (red dots) with respect to the MUSE field in WFM, shown here using the summed spectral slices within $4 \AA$ from $\lambda_{4 \mathrm{LGSF}}$ and smoothed with a Gaussian kernel of 3 pixels in radius from a 60-s exposure. The LGSs are located 62 arc sec away from the field center (marked by a black cross) in a fixed altitude-azimuth orientation. The derotation of the MUSE field over time implies that the LGSs rotate around the field center following the parallactic angle $\eta$, with their final position (in this specific case) marked with an empty red dot. The size of the out-of-focus LGS donuts is marked using dashed red circles. Black arrows indicate the orientation of the laser beam from each LGS, pointing away from the MUSE field. As a size reference, the central circle is 20 arc sec in radius.

$1 \times 1$-square-arc-min field of view of MUSE in WFM-N (see Fig. 4). At the laser wavelength $\lambda_{4 \mathrm{LGSF}}$, this observation reveals the out-of-focus LGS and thus the UT4 pupil (in this case, prior to the installation of the DSM), as well as the uplink laser beam. At the mean wavelength of the rovibrational Raman lines, the same data set reveals a clear view of the laser beam but not of the LGS donut. The LGS is created through the excitation of $\mathrm{Na}$ atoms at about $90 \mathrm{~km}$ of altitude, so the lack of Raman emission associated with the LGS itself is to be expected. We note that the laser beam is not continuously visible (either through Rayleigh or Raman scattering) all the way to the sodium layer. Using a model of the UT4 telescope and 4LGSF launch telescope (see the Appendix A for details), we find that the Rayleigh and Raman emission from the laser beam is undetected in our observations for altitudes beyond $30 \pm 5 \mathrm{~km}$ above ground, consistent with atmospheric LIDAR observations [49,50] and typical atmospheric density profiles [51].

Although not representative of normal operations, the on-axis observation of one of the LGSs and its associated uplink beam with MUSE allows the construction of a high signal-to-noise view of the Raman rovibrational lines associated with $\mathrm{O}_{2}$ and $\mathrm{N}_{2}$. The mean spectrum of the regions $\mathrm{R}_{1}-\mathrm{R}_{0}$ (each $6 \times 6$ square arc sec in size)

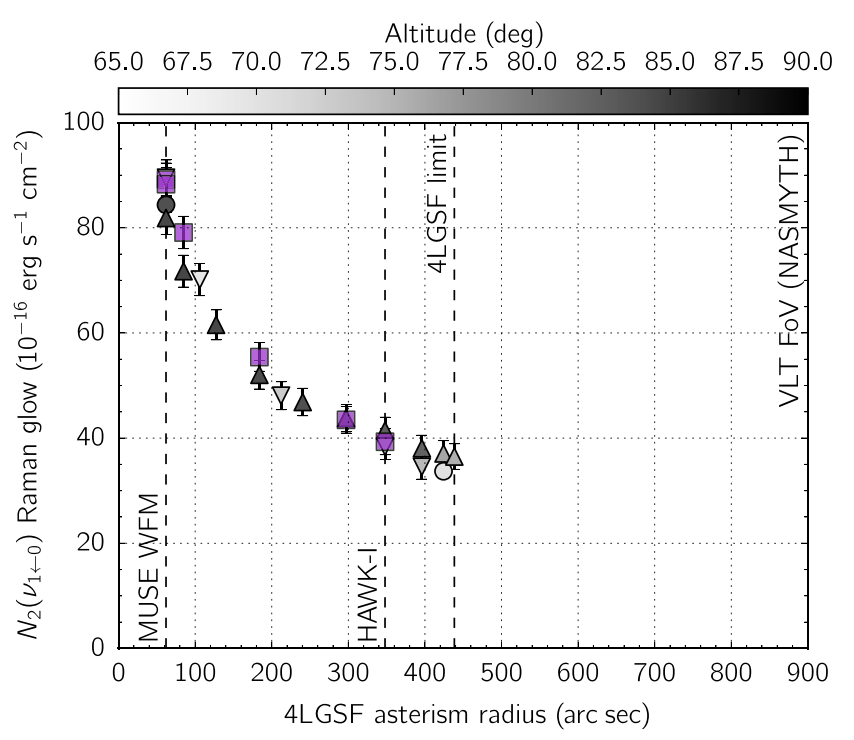

FIG. 3. Variation of the intensity of the Raman glow [measured via the flux of the $\mathrm{N}_{2}\left(\nu_{1 \leftarrow 0}\right)$ main vibrational line] within 20 arc sec of the center of the MUSE WFM field, for different 4LGSF asterism sizes. Different symbols denote distinct series of observations at different azimuths and times. Each grey symbol is color-coded as a function of the telescope altitude at the time of the observation (pre-GALACSI installation). Measurements acquired on April 15, 2017, post-GALACSI installation, are shown with purple squares. Error bars indicate the $3 \sigma$ uncertainties.

presented in Fig. 4 is built to that end. Following Ref. [47], the Raman spectral shifts of the rovibrational lines $\left.\Delta \nu_{1 \leftarrow 0}\right|_{S}$ (for the $S$ branch), $\left.\Delta \nu_{1 \leftarrow 0}\right|_{O}$ (for the $O$ branch), and $\left.\Delta \nu_{1 \leftarrow 0}\right|_{Q}$ (for the $Q$ branch) for a vibrating and rotating homonuclear diatomic molecule are

$\left.\Delta \nu_{1 \leftarrow 0}\right|_{S}=\Delta \nu_{0}+6 B_{1}+\left(5 B_{1}-B_{0}\right) J+\left(B_{1}-B_{0}\right) J^{2}$,

$\left.\Delta \nu_{1 \leftarrow 0}\right|_{Q}=\Delta \nu_{0}+\left(B_{1}-B_{0}\right) J+\left(B_{1}-B_{0}\right) J^{2}$,

with $J \in[0,1,2, \ldots]$, and

$\left.\Delta \nu_{1 \leftarrow 0}\right|_{O}=\Delta \nu_{0}+2 B_{1}-\left(3 B_{1}+B_{0}\right) J+\left(B_{1}-B_{0}\right) J^{2}$,

with $J \in[2,3,4, \ldots]$. Note that $B_{i}$ is the rotational constant of the diatomic molecule at the vibrational level $i$, which can be approximated as

$$
B_{i}=B_{e}-\alpha_{e}(i+0.5),
$$

with $B_{e}$ the rotational constant in equilibrium position and $\alpha_{e}$ the first-order term. From Refs. [52,53] and the references therein,

$$
\begin{aligned}
& B_{e, \mathrm{O}_{2}}=1.44562 \mathrm{~cm}^{-1}, \quad \alpha_{e, \mathrm{O}_{2}}=0.0159305 \mathrm{~cm}^{-1}, \\
& B_{e, \mathrm{~N}_{2}}=1.998241 \mathrm{~cm}^{-1}, \quad \alpha_{e, \mathrm{~N}_{2}}=0.0117318 \mathrm{~cm}^{-1} .
\end{aligned}
$$



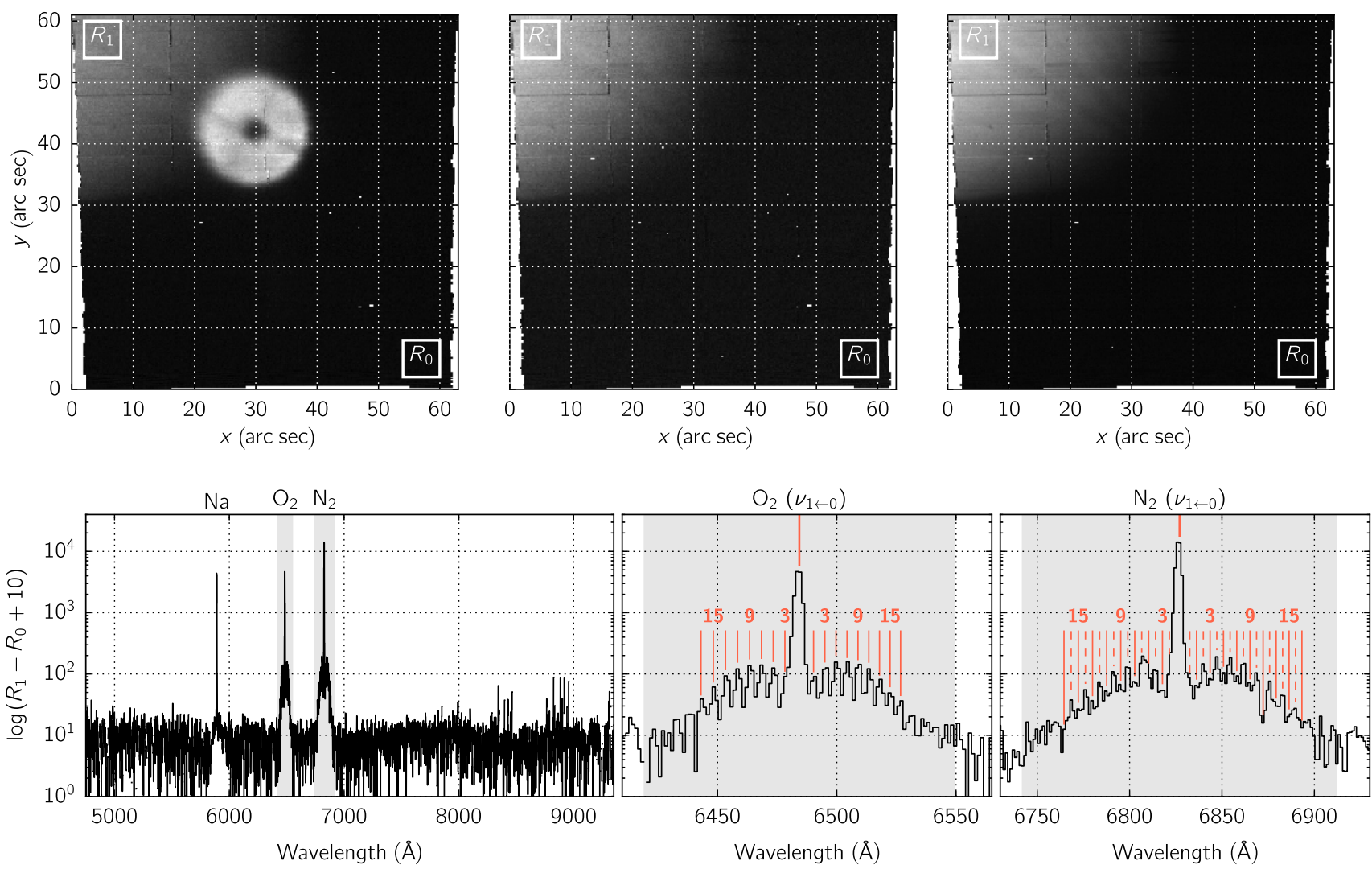

FIG. 4. Top panels: Summed MUSE spectral slices within $4 \AA$ from the laser line at $5889.959 \AA$ (left), the $\mathrm{O}_{2}$ Raman vibrational line at $6484.39 \AA$ (middle), and the $\mathrm{N}_{2}$ Raman vibrational line at $6827.17 \AA$ (right), with one of the LGS units voluntarily placed within the field of view. Both the out-of-focus donut of the LGS (emitted at the sodium layer altitude of about $90 \mathrm{~km}$ ) and the laser uplink beam (via Rayleigh and Mie scattering) are visible through the notch filter at the laser wavelength. At the Raman wavelengths, only the laser beam is visible. Bottom panels: Difference between the in-beam and out-beam MUSE spectra extracted from the regions $R_{1}$ and $R_{0}$ defined in the top panels. Only three features clearly dominate the residual noise: the laser line at $\lambda_{4 \mathrm{LGSF}}$ and the rovibrational Raman lines associated with the $\mathrm{O}_{2}$ and $\mathrm{N}_{2}$ molecules. For $\mathrm{O}_{2}$, red vertical lines show the predicted locations of the rotational sidelines for $J \in[1,3,5,7, \ldots, 17]$. For $\mathrm{N}_{2}$, red vertical lines show the predicted locations of the rotational sidelines for $J \in[0,1,2,3, \ldots, 17]:$ solid and dashed lines for odd and even values of $J$ (respectively).

The spectral location of the observed rotational sidebands (visible up to rotational quantum numbers $J=17$ in Fig. 4) are in perfect agreement with the theoretical predictions of the Raman shifts of the $S$ and $O$ branches, while the $Q$ branch remains unresolved by MUSE. We also note that for $\mathrm{O}_{2}$, only rotational sidelines for odd values of $J$ are visible, while for $\mathrm{N}_{2}$, rotational sidelines for even values of $J$ are stronger than for odd values of $J[47,54]$.

\section{EXTENDING THE SEARCH FOR RAMAN SCATTERING BEYOND THE $\nu_{1 \leftarrow 0}$ FUNDAMENTAL MODE OF $\mathrm{O}_{2}$ AND $\mathrm{N}_{2}$ MOLECULES}

Virtually all of the Rayleigh, Mie, and Raman scattering of laser photons occurs within the footprint of the uplink beams: narrow cylinders of $30 \mathrm{~cm}$ in diameter extending from the laser launch telescopes up the sodium layer at an altitude of about $90 \mathrm{~km}$ and beyond. As illustrated in Fig. 4, looking directly at the uplink beam with MUSE offers an ideal way of boosting our detection ability for the Raman scattering signatures of different molecules. Beyond $\mathrm{N}_{2}$ and $\mathrm{O}_{2}$ that make up (by volume) $78.09 \%$ and $20.95 \%$ of air, $\mathrm{CO}_{2}(0.04 \%), \mathrm{H}_{2} \mathrm{O}$ (variable, typically $<1 \%$ ), and $\mathrm{CH}_{4}$ (about $0.0002 \%$ ) are the next-best candidates for triggering laser-induced Raman scattering events in the sky of Cerro Paranal, given the clean air of the surrounding Atacama desert.

To further increase the signal of Raman-scattered photons compared to the observations described in Fig. 4 (with one LGS within the MUSE field of view), we acquired an additional series of observations with MUSE in which we crossed the laser beams over the field of view. One must stress once more that this is a setup that is clearly not representative of normal science operations. We performed these observations in four individual steps:

(1) Set up the 4LGSF asterism to the MUSE WFM size (62 arc sec in radius).

(2) Stop the propagation of all lasers but LGSU2, and place its LGS at the location of LGSU4. 

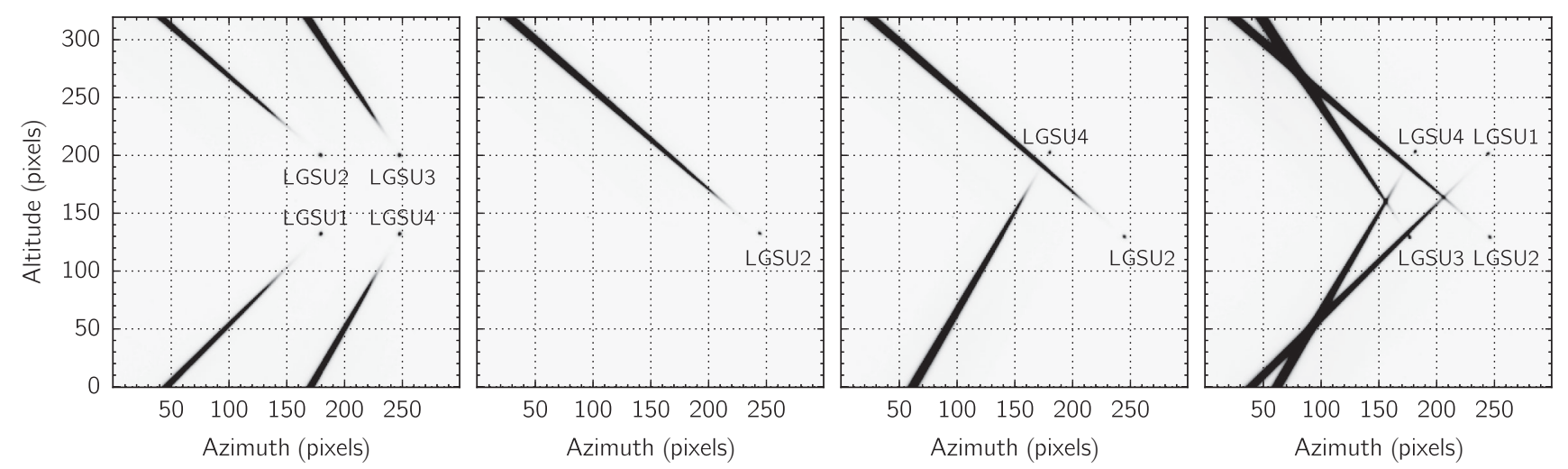

FIG. 5. Images from the LPC (1.29 arseconds per pixel) [55,56] with (from left to right) the 4LGSUs in their nominal MUSE-WFM asterism (radius of about $62 \mathrm{arc} \mathrm{sec),} \mathrm{LGSU2} \mathrm{at} \mathrm{the} \mathrm{position} \mathrm{of} \mathrm{LGSU4,} \mathrm{LGSU2} \mathrm{and} \mathrm{LGSU4} \mathrm{at} \mathrm{each} \mathrm{other's} \mathrm{default} \mathrm{positions,} \mathrm{and} \mathrm{all}$ LGSU positions swapped (LGSU1 $\rightarrow$ LGSU3, LGSU2 $\rightarrow$ LGSU4, LGSU3 $\rightarrow$ LGSU1, and LGSU4 $\rightarrow$ LGSU2). The off-axis location of the LPC (on the UT4 top ring) gives rise to the peculiar perspective of the crossed configurations.

(3) Without modifying the position of LGSU2, start the propagation of LGSU4 and place its LGS at the original location of LGSU2.

(4) Repeat the same procedure for the LGSUs 1 and 3.

This process is illustrated in Fig. 5 by means of images from the laser positioning camera (LPC) $[55,56]$ located on

the top ring of UT4. The unusual geometry of the beams in the crossed configurations is a direct consequence of the off-axis location of this camera. The lack of a single intersection point for the four laser beams is due to the rectangular distribution of the launch telescopes, compared to the square shape of the LGS asterism. From the MUSE
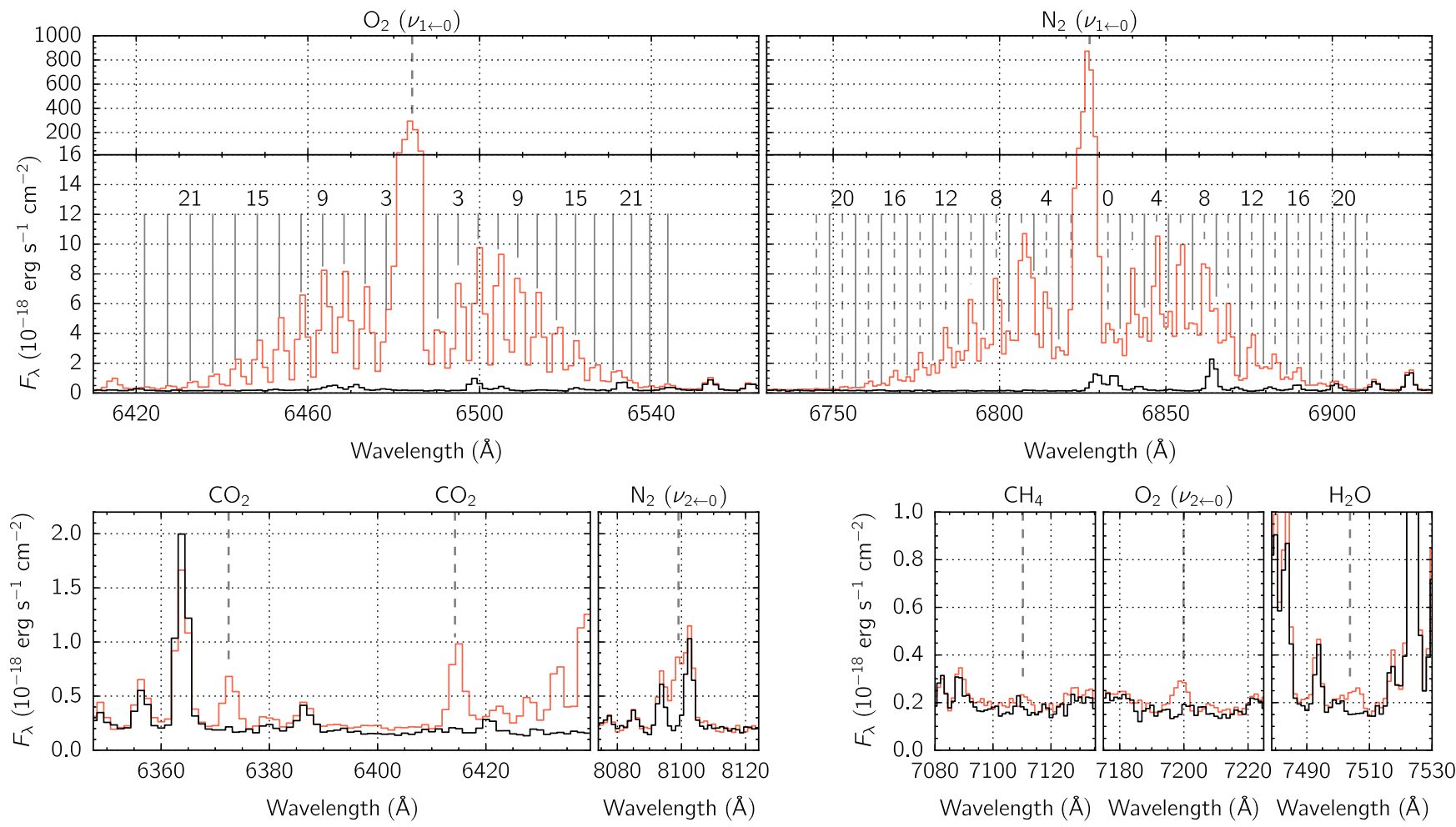

FIG. 6. Mean spectra from MUSE spaxels located within 20 arc sec from the center of the field, with the four LGS beam crossed over the field (red), and with no laser propagating (black). Each panel is centered on a Raman line of interest (which, unlike sky lines, have no counterpart in the reference spectrum in black). The rotational sidelines associated with the $\nu_{1 \leftarrow 0}$ transition from the $\mathrm{O}_{2}$ and $\mathrm{N}_{2}$ molecules are detected up to $J=23$. The fundamental Raman lines from $\mathrm{CO}_{2}$ and $\mathrm{H}_{2} \mathrm{O}$ are clearly visible when the lasers are propagating in this configuration. The first overtone associated with the $\nu_{2 \leftarrow 0}$ transition from $\mathrm{O}_{2}$ and $\mathrm{N}_{2}$ is also detected (the latter between two sky lines). 
perspective, this implies that the different beams will together excite several altitude layers of the atmosphere below $10 \mathrm{~km}$ within the instrument's WFM field of view of 1 square arc min.

The mean spectra extracted from all the spatial pixels (spaxels) located within 20 arc sec from the center of the MUSE WFM field of view are presented in Fig. 6, both for a 60-s exposure with the four laser beams crossed and a subsequent reference 60-s exposure with no laser propagating. The Raman lines associated with the fundamental $\nu_{1 \leftarrow 0}$ rovibrational excitation of the $\mathrm{O}_{2}$ and $\mathrm{N}_{2}$ molecules are clearly detected, with rotational sidebands visible up to $J=23$ [see Eqs. (1) and (2)].

Beyond the fundamental Raman lines, we also detect the first overtone lines (corresponding to the $\nu_{2 \leftarrow 0}$ transition) [57-59] from $\mathrm{O}_{2}$ and $\mathrm{N}_{2}$ molecules at $7200.02 \AA$ $\left(\Delta \nu_{0, \mathrm{O}_{2}}^{\prime}=3089.2 \mathrm{~cm}^{-1}\right) \quad$ and $8099.23 \AA \quad\left(\Delta \nu_{0, \mathrm{~N}_{2}}^{\prime}=\right.$ $4631.2 \mathrm{~cm}^{-1}$ ). These second-order lines are 3-4 orders of magnitude fainter than the peak intensity of the fundamental vibrational line of $\mathrm{N}_{2}$.

At similar intensity levels, we unambiguously detect the presence of Raman scattering from $\mathrm{CO}_{2}$ molecules $\left[\Delta \nu_{0, \mathrm{CO}_{2}}=1285.8 \mathrm{~cm}^{-1} ; 1388.1 \mathrm{~cm}^{-1}\right]$ at $6372.57 \AA$ and $6414.39 \AA[60]$ and $\mathrm{H}_{2} \mathrm{O}$ molecules $\left[\Delta \nu_{0, \mathrm{H}_{2} \mathrm{O}}=\right.$ $\left.3651.7 \mathrm{~cm}^{-1}\right]$ at $7503.93 \AA[60,61]$. We note that these observations were acquired with low levels of precipitable water vapor (about $1 \mathrm{~mm}$ ) and humidity (about 10\%). The signature of Raman scattering from $\mathrm{CH}_{4}$ molecules $\left[\Delta \nu_{0, \mathrm{CH}_{4}}=2914.2 \mathrm{~cm}^{-1}\right]$ at $7110.43 \AA[60]$ is only tentatively detected with a signal-to-noise ratio of $\mathrm{S} / \mathrm{N}<3$.

\section{QUANTIFYING THE INTENSITY OF LASER-INDUCED RAMAN LINES}

Predicting the exact impact of Raman scattering from a LGS system on a given astronomical observation is not straightforward. The number of laser photons that are subject to Raman scattering is proportional both to the (on-sky) power of the laser system employed and the density of molecules within the footprint of the uplink beam, which varies as a function of the altitude. In addition, the intensity of the contamination for any given telescope at any given time also depends on the collision parameters (i.e., the scattering angle and the distance to the impacting portion of the uplink laser beam from the telescope). Finally, the intensity of the Raman contamination also depends heavily on the characteristics of the instrument involved: for example, the central wavelength and width of the filter for an imager, and the spectral range and resolution for a spectrograph. The specificities of the telescope (including the overall throughput and aperture) also play a role. Altogether, this implies that it is very difficult to summarize with precision the impact of laserinduced Raman scattering for all possible instruments, telescopes, LGS systems, and observatories.

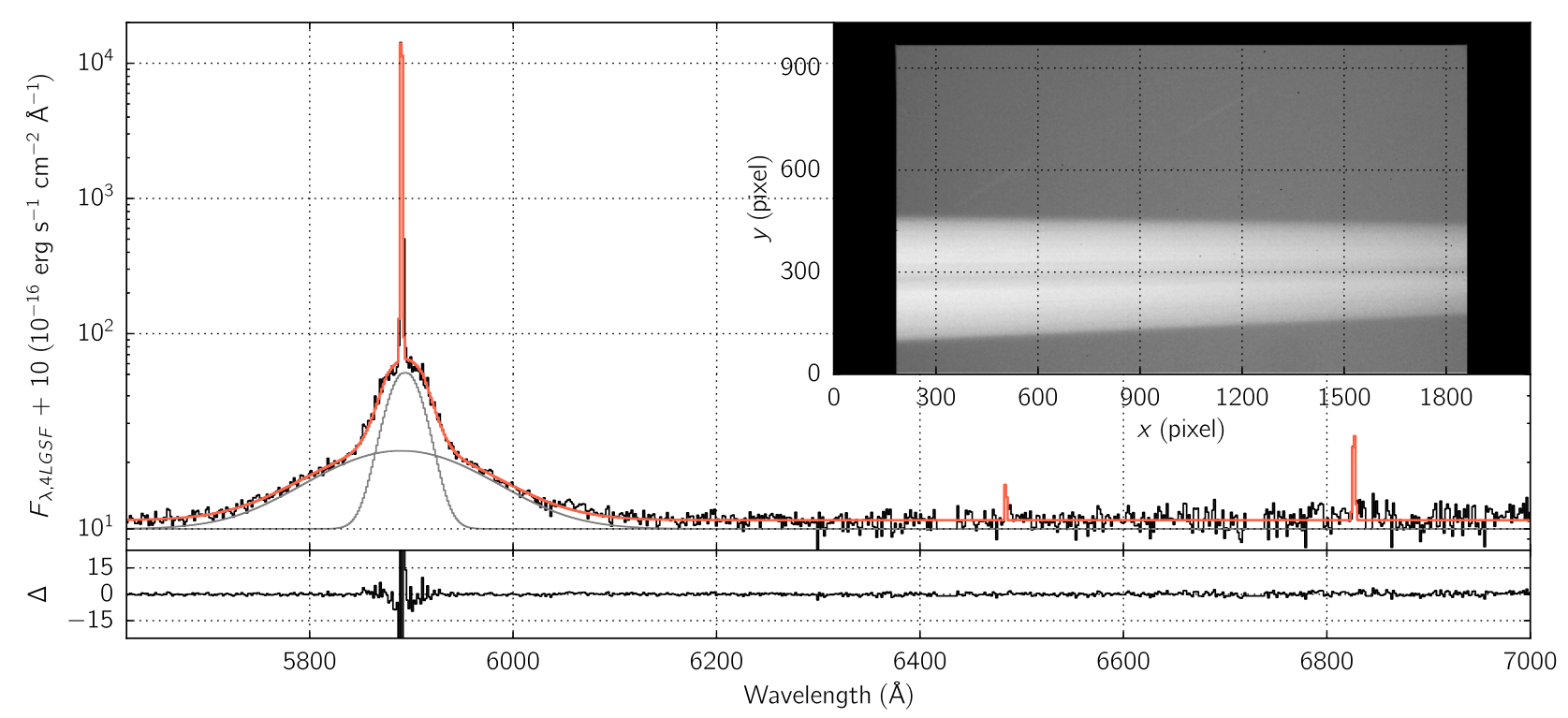

FIG. 7. FORS2 spectra of the uplink beam of LGSU1 from the 4LGSF at an altitude of $25 \mathrm{~km}$ above ground (black), and our fit to the spectra (red) including the laser line and the $\mathrm{N}_{2}\left(\nu_{1 \leftarrow 0}\right)$ and $\mathrm{O}_{2}\left(\nu_{1 \leftarrow 0}\right)$ Raman lines. The main laser line at $\lambda_{4 \mathrm{LGSF}}$ requires three Gaussian components to be properly reproduced. The narrowest component corresponds to the laser photons subject to elastic Rayleigh and Mie scattering. The intermediate Gaussian component corresponds to inelastic rotational Raman scattering and is in fact a superposition of multiple narrow lines associated with different molecules. The broadest component is an instrumental artifact of FORS2. Top right: R-band image from chip 1 of FORS2 acquired at the same location, with the uplink beam (horizontal on the image) clearly visible. The running shadow of the secondary mirror of UT1 makes the innermost region of the laser beam darker. Star trails are visible in the background, given that UT1 was idle during the exposure. 
TABLE I. Integrated fluxes for the different laser-induced Raman lines from the uplink beam at an altitude of $27.6 \mathrm{~km}$ above sea level, normalized to the power of a (continuous-wave) 1 -W laser shining at $5889.959 \AA$. Uncertainties on the quoted fluxes are $\lesssim 15 \%$. For the fundamental Raman lines of $\mathrm{N}_{2}$ and $\mathrm{O}_{2}$, the fluxes refer to the central peak only (i.e., the $Q$ branch). The flux associated with the main Rayleigh line of the laser in the same observational setup is also indicated.

\begin{tabular}{|c|c|c|c|c|c|c|c|c|c|}
\hline Raman line & $\lambda_{4 \mathrm{LGSF}}$ & $\mathrm{CO}_{2}$ & $\mathrm{CO}_{2}$ & $\mathrm{O}_{2}\left(\nu_{1 \leftarrow 0}\right)$ & $\mathrm{N}_{2}\left(\nu_{1 \leftarrow 0}\right)$ & $\mathrm{CH}_{4}$ & $\mathrm{O}_{2}\left(\nu_{2 \leftarrow 0}\right)$ & $\mathrm{H}_{2} \mathrm{O}$ & $\mathrm{N}_{2}\left(\nu_{2 \leftarrow 0}\right)$ \\
\hline Raman shift $\left(\mathrm{cm}^{-1}\right)$ & . & 1285.8 & 1388.1 & 1556.4 & 2330.7 & 2914.2 & 3089.2 & 3651.7 & 4631.2 \\
\hline$\lambda_{\text {obs }}(\AA)$ & 5889.959 & 6372.57 & 6414.39 & 6484.39 & 6827.17 & 7110.43 & 7200.02 & 7503.93 & 8099.23 \\
\hline Flux $\left(10^{-20} \mathrm{erg} \mathrm{s}^{-1} \mathrm{~cm}^{-2} \mathrm{~W}^{-1}\right)$ & $1.9 \times 10^{7}$ & 11.3 & 18.9 & $6.8 \times 10^{3}$ & $2.0 \times 10^{4}$ & $\lesssim 1.1$ & 3.2 & 2.7 & 16.1 \\
\hline
\end{tabular}

These factors notwithstanding, we provide in Table I indicative fluxes of the different Raman lines identified in Sec. III. These fluxes are scaled to the brightness of the uplink beam of a (continuous-wave) 1-W laser guide star with $\lambda_{\text {LGS }}=\lambda_{4 \text { LGSF }}$ observed at an altitude of $25 \mathrm{~km}$ above ground on Cerro Paranal, equivalent to $27.6 \mathrm{~km}$ above sea level. The appropriate scaling for the different line fluxes (measured from the spectrum presented in Fig. 6) was derived using dedicated observations of one of the 4LGSF uplink beams using the FORS2 [62] spectrograph mounted on the Cassegrain focus of UT1 at the VLT, performed during the evening twilight of November 28, 2016, under clear conditions. During the test, UT4 was pointed directly away from UT1, with LGSU1 (alone) propagating in center field at an altitude of $89.000^{\circ}$. UT1, located at a (Vincenty) distance of $130.22 \mathrm{~m}$ from UT4, was pointed directly towards UT4 at an altitude of $88.694^{\circ}$. In this configuration (with both telescopes idle during the entire duration of the test), UT1 was effectively pointing towards the uplink laser beam at an altitude of $25 \mathrm{~km}$ above ground.

The resulting FORS2 long-slit spectrum for a 60-s exposure acquired with a slit width of 0.28 arc sec and the $600 \mathrm{RI}+19$ grism is presented in Fig. 7. The data were processed using the ESO Reflex [63] workflow (v 5.0.20) for FORS2. We use Gaussian components to fit the main laser line and the fundamental Raman lines associated with $\mathrm{O}_{2}$ and $\mathrm{N}_{2}$ molecules, combined with a constant level to account for the existing continuum residual post-sky subtraction. We note that fitting the main laser line at $\lambda_{4 \mathrm{LGSF}}$ requires three Gaussian components. The narrowest (unresolved) component is the brightest and corresponds to the laser photons that experienced elastic Rayleigh and Mie scattering. The intermediate component, with a full width at half maximum (FWHM) of $44.2 \pm 0.6 \AA$, corresponds to the laser photons that experienced Raman scattering and excited the rotational mode of molecules (as opposed to the rovibrational modes described until now). This component is, as such, a collection of narrow emission lines-blended at the spectral resolution of FORS2 of $R \cong 2900$ - associated with the different air molecules present in the footprint of the laser beam. The existence of (some of) these narrow emission lines is best seen in the fit residual $\Delta$. Finally, the broadest Gaussian component associated with the main laser line is an instrumental artifact of the FORS2 spectrograph. A similar glow is present, for example, around the brightest lines nearing saturation in arc lamp exposures. The exact origin of this glow is under investigation by the FORS2 instrument team.

We derive the appropriate scaling factor for all the Raman line fluxes presented in Table I by comparing the FORS2 observations of the $\mathrm{N}_{2}$ and $\mathrm{O}_{2}$ fundamental Raman lines with the MUSE observations described in Sec. III. The resulting fluxes thus correspond to the integrated flux of a single laser uplink beam observed at an altitude of $25 \mathrm{~km}$, under the (reasonable) assumption that the composition of the atmosphere above Cerro Paranal does not vary significantly up to heights of about $25 \mathrm{~km}$ above ground (i.e., the intensity ratio between the different laser-induced Raman lines is constant).

The line fluxes provided in Table I are intended for observatories and their users to assess the possible impact of laser-induced Raman scattering on regular operations. We stress, however, that these fluxes must be treated with caution. They provide a realistic measure of the different Raman line fluxes, but their validity is limited to the meteorological specificities of a given site and seasonal variations in the temperature profile and water vapor content of the atmosphere. For reference and comparison purposes, the IR temperature of the sky was $(-88 \pm 5){ }^{\circ} \mathrm{C}$ during the FORS2 observations, with a temperature at ground level of $(16 \pm 1){ }^{\circ} \mathrm{C}$. For the $\mathrm{H}_{2} \mathrm{O}$ line, in particular, the precipitable water vapor during the (scaled) MUSE observations was about $1 \mathrm{~mm}$ and the humidity about $10 \%$ at ground level.

\section{IMPLICATIONS OF LASER-INDUCED RAMAN SCATTERING FOR ASTROPHYSICAL OBSERVATIONS}

Although the physics involved is elegant, the possible contamination of observations via laser-induced rovibrational Raman scattering may become a clear concern from the operational perspective of an astronomical observatory. As illustrated in Fig. 4, rovibrational Raman scattering leads to spectrally complex and broad structures in optical regions containing important astrophysical spectral lines (see Fig. 8). Although the structure of the Raman lines associated with diatomic molecules like $\mathrm{N}_{2}$ and $\mathrm{O}_{2}$ is well known, removing these lines from the data a posteriori will necessarily induce some residual photon noise affecting the 


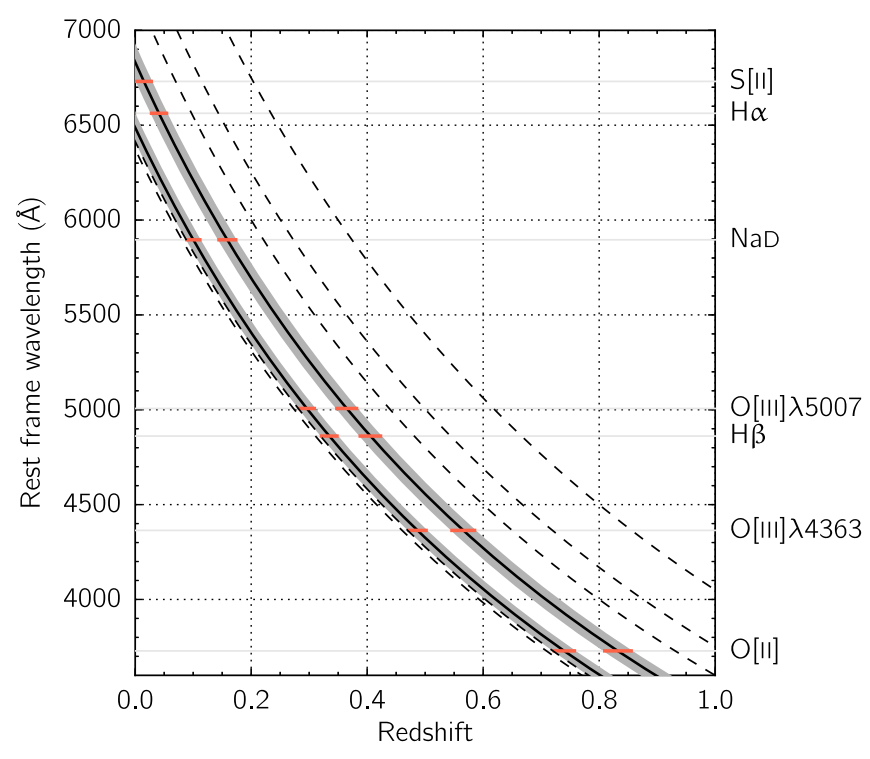

FIG. 8. Spectral region-in the rest frame of an astrophysical source-affected by laser-induced Raman contamination as a function of redshift, assuming $\lambda_{\text {LGS }}=589 \mathrm{~nm}$. For both $\mathrm{O}_{2}$ and $\mathrm{N}_{2}$ molecules, the greyed-out zone marks the spectral extent of the rotational sidelines out to $J=17$. The redshift zones associated with a few important optical lines are highlighted in red. Spectral regions affected by the (3-4 orders of magnitude fainter) Raman contamination from $\mathrm{CO}_{2}$ molecules, $\mathrm{H}_{2} \mathrm{O}$ molecules, and the first overtone of $\mathrm{O}_{2}$ molecules and $\mathrm{N}_{2}$ molecules are indicated with dashed-dotted lines.

quality of observations, in particular, for deep fields and/or extremely faint targets. The intensity of the Raman rovibrational lines is also temperature dependent $[49,64]$ and thus potentially time dependent. The use of notch filters, such as in the case of MUSE, is only viable (from a scientific efficiency perspective) to alleviate the impact of the main Rayleigh line and rotational Raman lines in its immediate surroundings. It is thus crucial to handle the impact of rovibrational Raman contamination by first ensuring the proper baffling of the instrument, in addition to designing appropriate calibrations, observation procedures, and datareduction pipelines.

Certainly, the existence of laser-induced Raman scattering is not unique to Cerro Paranal, but it is common to any astronomical observatory employing similar devices. The majority (today) of astronomical telescopes rely on 589-nm lasers, but the emergence of so-called Rayleigh LGS systems with shorter lasing wavelengths implies that at specific sites, laser-induced Raman scattering will affect different spectral regions. For completeness, we list in Table II the wavelengths of the atmospheric rovibrational Raman lines described in Sec. III as a function of the laser wavelength $\lambda_{\mathrm{LGS}}$ for the main astronomical telescopes equipped with a LGS system. The efficiency of Raman scattering increases at shorter wavelengths, so Rayleigh LGS systems are likely to be more strongly affected by it, modulo the on-sky power of the lasers involved.
In most cases, LGS systems are used to feed (near-)IR instruments, oblivious to the existence of Raman-scattered photons by the $\mathrm{O}_{2}$ and $\mathrm{N}_{2}$ molecules (which are the most abundant in the atmosphere and thus give rise to the strongest signal). Yet, the presence of laser-induced Raman-scattered photons can also affect other nearby telescopes and instruments at sites hosting multiple telescopes, such as Mauna Kea or Cerro Paranal. The distance between the different telescopes is a key factor influencing the frequency of so-called laser collisions. For the case of Cerro Paranal, the probability of at least one collision between the uplink laser beams from UT4 and any of the UTs or the VLT Survey Telescope (VST) $[80,81]$ is estimated to be greater than $90 \%$ on any given night [82]. To avoid the accidental contamination of laser-sensitive observations at nearby telescopes, a laser-collision prediction tool was initially developed for the W.M. Keck LGS system [83] and eventually ported to the observatory of La Palma [84] and Cerro Paranal [36]. One essential component of this tool is that the different telescopes on any given site must declare themselves as being laser sensitive or not. On Cerro Paranal, the sensitivity of the different instruments mounted on all the UTs was assessed individually for each system, filter set, grating, and grism, depending on whether a given setup can see (or not) the main laser Rayleigh line at $589 \mathrm{~nm}$. In other words, the system has so far been oblivious to the possibility of rovibrational Raman contamination. In particular, laser collisions for instrumental setups sensitive only to the rovibrational Raman lines (but not the main laser line) do not trigger any red flag. As discussed in Sec. IV, each instrument will be affected by rovibrational Raman contamination at a different level. Broadband imagers are expected to be less impacted than high-resolution spectrographs, and each case must undoubtedly be assessed individually. Currently, the individual Raman sensitivity assessment for all affected instruments on Cerro Paranal is underway.

With respect to the next generation of extremely large telescopes, our observations of laser-induced Raman scattering on Cerro Paranal will be particularly applicable to the HARMONI [72] visible and near-IR integral field spectrograph to be installed as a first-light instrument on the Extremely Large Telescope (ELT) at Cerro Armazones. HARMONI will be fed corrected wavefronts by the ELT ATLAS LGS system [85], which relies on six 589-nm lasers similar to those of the 4LGSF (according to the current design). HSIM [86] is dedicated software designed to simulate observations with HARMONI. Thus, this tool likely offers the ideal means for (1) evaluating the impact of laser-induced Raman scattering on HARMONI observations and (2) implementing suitable observing strategies.

Finally, the 4LGSF lasers and associated laser-induced Raman emission are currently under (ongoing) close scrutiny, including via dedicated observations using the VST and FLAMES [87] on UT2, as well as with the Gran 
TABLE II. Wavelength of the main atmospheric, laser-induced Raman scattering lines as a function of the LGS wavelength $\lambda_{\text {LGS }}$ at different astronomical telescopes.

\begin{tabular}{|c|c|c|c|c|c|c|c|c|c|c|}
\hline Telescope & LGS system & $\begin{array}{l}\lambda_{\mathrm{LGS}} \\
{[\mathrm{nm}]}\end{array}$ & $\begin{array}{l}\mathrm{CO}_{2} \\
{[\mathrm{~nm}]}\end{array}$ & $\begin{array}{l}\mathrm{CO}_{2} \\
{[\mathrm{~nm}]}\end{array}$ & $\begin{array}{c}\mathrm{O}_{2}\left(\nu_{1 \leftarrow 0}\right) \\
{[\mathrm{nm}]}\end{array}$ & $\begin{array}{c}\mathrm{N}_{2}\left(\nu_{1 \leftarrow 0}\right) \\
{[\mathrm{nm}]}\end{array}$ & $\begin{array}{l}\mathrm{CH}_{4} \\
{[\mathrm{~nm}]}\end{array}$ & $\begin{array}{c}\mathrm{O}_{2}\left(\nu_{2 \leftarrow 0}\right) \\
{[\mathrm{nm}]}\end{array}$ & $\begin{array}{l}\mathrm{H}_{2} \mathrm{O} \\
{[\mathrm{nm}]}\end{array}$ & $\begin{array}{c}\mathrm{N}_{2}\left(\nu_{2 \leftarrow 0}\right) \\
{[\mathrm{nm}]}\end{array}$ \\
\hline Gemini North & ALTAIR $[8,9]$ & & & & & & & & & \\
\hline Gemini South & GeMS $[10-12]$ & & & & & & & & & \\
\hline W. M. Keck & LGSAO $[6,7]$ & & & & & & & & & \\
\hline Lijang 1.8-m Telescope & LGS-AOS [65] & & & & & & & & & \\
\hline Shane 3-m Telescope & ShaneAO $[66,67]$ & & & & & & & & & \\
\hline Subaru & AO188 $[13,14]$ & 589 & 637 & 641 & 648 & 683 & 711 & 720 & 750 & 810 \\
\hline VLT (UT4) & $4 \mathrm{LGSF}[16,20]$ & & & & & & & & & \\
\hline VLT (UT4) & PARLA $[15]$ & & & & & & & & & \\
\hline Thirty Meter Telescope & LGSF $[68,69]$ & & & & & & & & & \\
\hline Giant Magellan Telescope & GMT AO [70] & & & & & & & & & \\
\hline Extremely Large Telescope & ATLAS \& MAORY $[71-74]$ & & & $\mid$ & $\mid$ & $\mid$ & $\mid$ & | & $\mid$ & | \\
\hline Large Binocular Telescope & ARGOS $[75,76]$ & 532 & 571 & 574 & 580 & 607 & 630 & 637 & 660 & 706 \\
\hline William Hershel Telescope & GLAS $[77]$ & 515 & 552 & 555 & 560 & 585 & 606 & 612 & 634 & 676 \\
\hline Hale 5-m Telescope & PULSE [78] & 1 & । & 1 & I & | & । & । & | & | \\
\hline $\begin{array}{c}\text { Southern Astrophysical } \\
\text { Research Telescope }\end{array}$ & SAM [79] & $\begin{array}{c}355 \\
\mid\end{array}$ & $\begin{array}{c}372 \\
\mathrm{I}\end{array}$ & $\begin{array}{c}373 \\
\text { I }\end{array}$ & $\begin{array}{c}376 \\
\text { I }\end{array}$ & $\begin{array}{c}387 \\
1\end{array}$ & $\begin{array}{c}396 \\
\text { I }\end{array}$ & $\begin{array}{c}399 \\
\text { I }\end{array}$ & $\begin{array}{c}408 \\
\mid\end{array}$ & $\begin{array}{c}425 \\
1\end{array}$ \\
\hline
\end{tabular}

Telescopio Canarias using the Wendelstein Laser Guide Star [88]. Combined with existing and upcoming MUSE and GALACSI tests, these data sets are intended to directly assess the impact of laser-induced Raman scattering on specific instruments on Cerro Paranal and at the Observatorio del Roque de los Muchachos.

\section{ACKNOWLEDGMENTS}

We thank the AOF Builders [20] for the construction and installation of the AOF on UT4 at the VLT. We are grateful to Alain Smette for stimulating discussions over the course of the commissioning tests; to George Hau, Julien Girard, Thomas Pfrommer, Steffen Mieske, Andreas Kaufer, Tim de Zeeuw, and Roland Bacon for their feedback on this article; to Claudia Reyes Saez, Claudia Cid, Susana Cerda, and Diego Parraguez for their operational support; and to Joe Anderson for his input regarding the behavior of the FORS2 spectrograph. We also thank the two anonymous referees for their constructive reviews. We used the following PYTHON packages in the research for this article: MATPLOTLIB [89]; ASTROPY, a community-developed core PYTHON package for Astronomy [90]; APLPY, an opensource plotting package for PYTHON [91], and MPFIT, a PYTHON script that uses the Levenberg-Marquardt technique [92] to solve least-squares problems, based on an original FORTRAN code that is part of the MINPACK-1 package. This research has also made use of the following: MONTAGE, funded by the National Science Foundation under Grant No. ACI-1440620 and previously funded by the National Aeronautics and Space Administration's Earth Science Technology Office, Computation Technologies Project, under Cooperative Agreement No. NCC5-626 between NASA and the California Institute of Technology; the ALADIN interactive sky atlas [93]; SAOIMAGE DS9 [94] developed by Smithsonian Astrophysical Observatory; and NASA's Astrophysics Data System. This work is based on observations made with ESO Telescopes at the La Silla Paranal Observatory. All the observations described in this article are freely available online from the ESO Data Archive. F. P. A. V., C. O. and Y. L. J. each acknowledge a Fellowship from the European Southern Observatory.

\section{APPENDIX: CONSTRAINING THE ALTITUDE OF RAMAN EMISSION WITH A TOY MODEL OF THE UT4 AND 4LGSF SYSTEMS}

The geometry and spatial extent of the Rayleigh emission from the laser beam visible in Fig. 4 is directly related to the altitude at which the scattering of the laser photons occurs (either via Rayleigh or Raman processes). The geometry of the problem is such that a simple model of the UT4 telescope and 4LGSF launch system can be used to constrain the altitude at which the laser beam is visible within the MUSE field of view. A schematic of the 4LGSF systems and UT4 is presented in Fig. 9, along with all the relevant quantities of our model. 


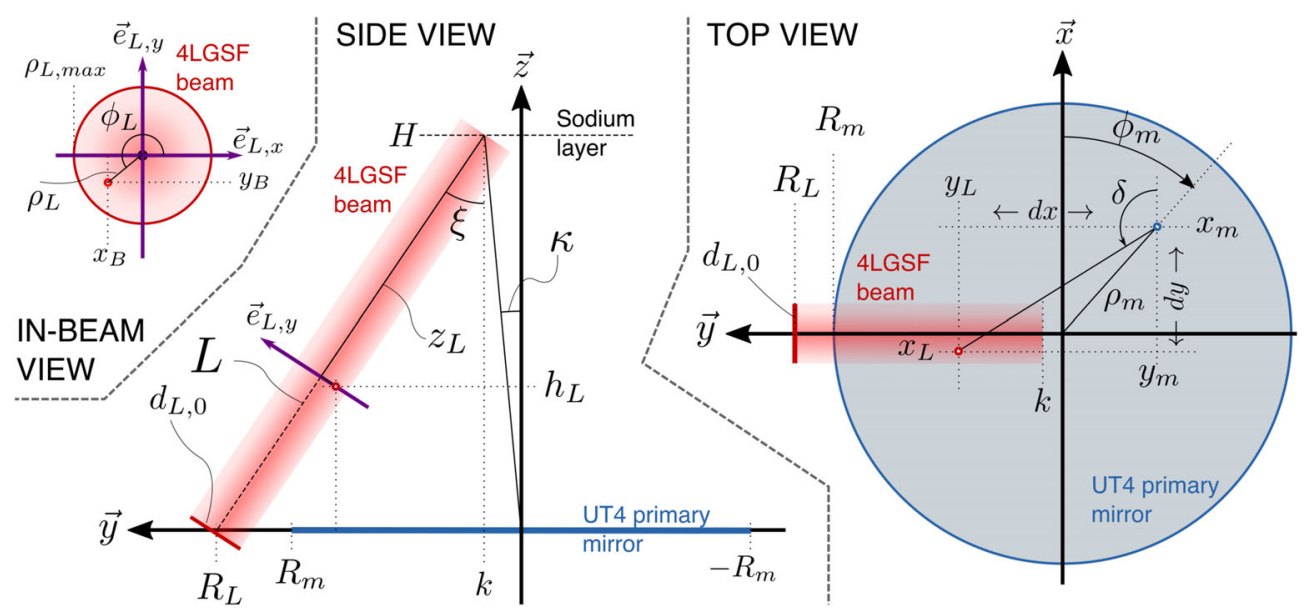

FIG. 9. Simple model of the 4LGSF (one laser only) and UT4 (reduced to a flat primary mirror). The laser is set to propagate from a distance $R_{L}$ from the optical axis of the telescope along the $\vec{y}$ axis. The beam is $30 \mathrm{~cm}$ in diameter and assumed to maintain a constant width as a function of altitude.

We define $R_{m}=4.1 \mathrm{~m}$, the radius of the primary mirror of UT4. Let us further assume that

$$
\begin{aligned}
H & =90000 \mathrm{~m}, \\
d_{L, 0} & =0.3 \mathrm{~m}, \\
R_{L} & =5.51 \mathrm{~m},
\end{aligned}
$$

where $H$ is the altitude of the 4LGSF guide star (i.e., the altitude of the sodium layer above the primary mirror), $d_{L, 0}$ is the diameter of the laser beam, and $R_{L}$ is the distance between the optical axis of UT4 and the location where the 4LGSF laser beam would cross the mirror plane (if extended backward from the launch telescope). We also assume that the laser beam (which is effectively a Gaussian beam) has a constant diameter as a function of the altitude and that the observation is performed at zenith.

We define $\kappa$ as the angle between the optical axis of UT4 and the location of the 4LGSF guide star, as measured from the center of the primary mirror. This angle corresponds to a physical offset projected onto the mirror surface $k$ :

$$
k=H \tan \kappa .
$$

When the laser guide star is placed at the center of the MUSE field of view, $\kappa=0 \Rightarrow k=0$. The total path length of the laser beam to the sodium layer $L$ is

$$
L=\sqrt{\left(R_{L}-k\right)^{2}+H^{2}} .
$$

To reconstruct the footprint of the laser beam in the MUSE field of view, we perform a crude ray-tracing exercise and construct the observed position (in the MUSE field of view) of all sky locations illuminated by the 4LGSF beam. Each location within the laser beam can be described with $z_{L}$, its distance to the sodium layer (along the beam propagation axis); $\rho_{L}$, its distance from the beam center; and $\phi_{L}$, its azimuth. In the subsequent analysis, we sample the laser beam at intervals of $1 \mathrm{~km}$ along its propagation axis, and each slice with 3 radial and 10 azimuthal steps. For a chunk of atmosphere illuminated by the laser with coordinates $\left(z_{L} ; \rho_{L} ; \phi_{L}\right)$, its projected coordinates in the mirror plane $\left(x_{L} ; y_{L}\right)$ are

$$
\begin{aligned}
& x_{L}=x_{B}, \\
& y_{L}=y_{B} \cos \xi+z_{L} \sin \xi+k,
\end{aligned}
$$

with

$$
\begin{aligned}
& x_{B}=\rho_{L} \cos \phi_{L}, \\
& y_{B}=\rho_{L} \sin \phi_{L} .
\end{aligned}
$$

Here, we assume a geometry in which the laser beam is launched from the $\vec{y}$ axis at the location $\left(0 ; R_{L}\right)$, with the laser $\vec{e}_{L, x}$ axis in the $\vec{x}-\vec{z}$ plane, the $\vec{e}_{L, y}$ axis in the $\vec{y}-\vec{z}$ plane, and the angle $\phi_{L}$ increasing counter-clockwise from the $\vec{e}_{L, x}$ axis. The angle $\xi$ is the angle between the vertical and the laser propagation axis as seen from the altitude $H$, i.e.,

$$
\tan \xi=\frac{R_{L}-k}{H}
$$

The altitude $h_{L}$ of the sky element located at $\left(z_{L} ; \rho_{L} ; \phi_{L}\right)$ can be written as

$$
\begin{aligned}
h_{L} & =H-z_{L} \cos \xi-y_{B} \sin \xi \\
& =H-z_{L} \cos \xi-\rho_{L} \sin \xi \sin \phi_{L}
\end{aligned}
$$

so the opening angle $\eta$ between the center of the MUSE field of view and the location on the sky of the said element is, as seen from the location $\left(x_{m} ; y_{m}\right)$ on the primary mirror, 

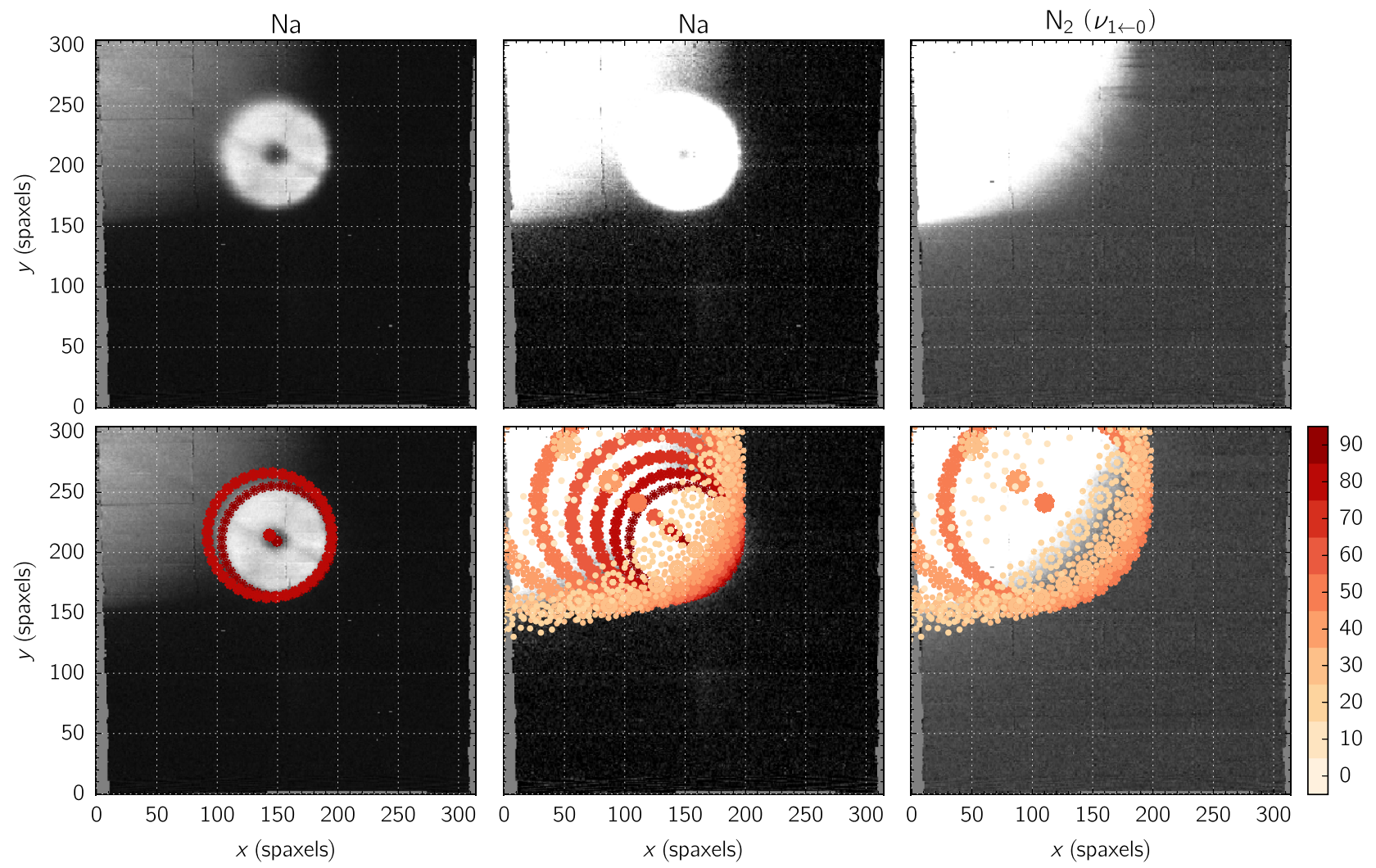

FIG. 10. Top panels: Summed MUSE slices within $4 \AA$ from $\lambda_{4 \mathrm{LGSF}}$ (left panel; middle panel: contrast enhanced) and $6826.73 \AA$ [ $\equiv \mathrm{N}_{2}$ $\left(\nu_{1 \leftarrow 0}\right)$; right panel] for an observation acquired with the LGS purposely located within the MUSE field of view. Bottom panels: Same as top, but with our modeled footprint of the laser beam as a function of the altitude overlaid (left panel: $80 \mathrm{~km}$ and $90 \mathrm{~km}$; middle panel: $0 \mathrm{~km}-90 \mathrm{~km}$; right panel: $10 \mathrm{~km}-50 \mathrm{~km}$ ). The out-of-focus LGS donut size is well reproduced by the model, with a diffuse extension to the top left approximately matched by the $85-\mathrm{km}$ layer, and consistent with the typical thickness of a few km of the sodium layer. The Rayleigh and Raman beams are best matched by the model out to altitudes of $30 \pm 5 \mathrm{~km}$ above ground. Each starlike pattern in the modeled footprint of the 4LGSF beam (at a given altitude) is associated with one of our chosen sampling locations on the primary mirror - which, taken altogether, trace the spatial extent of the unresolved beam. A movie of the modeled beam footprint sampling the altitude in steps of $1 \mathrm{~km}$ is available in Ref. [95].

$$
\eta=\frac{\pi}{2}-\arctan \left(\frac{h_{L}}{\sqrt{d x^{2}+d y^{2}}}\right)
$$

$$
\tan \delta=\frac{d y}{d x}
$$

with

$$
\begin{aligned}
& d x=x_{m}-x_{L}, \\
& d y=y_{m}-y_{L} .
\end{aligned}
$$

Finally, this can be translated to coordinates in the MUSE frame (in radians):

$$
\begin{aligned}
& x_{\mathrm{MUSE}}=\eta \cos \delta, \\
& y_{\mathrm{MUSE}}=\eta \sin \delta,
\end{aligned}
$$

with $\delta$ the azimuth (measured in the mirror plane) between the location $\left(x_{m} ; y_{m}\right)$ and the projected chunk of atmosphere at $\left(x_{L} ; y_{L}\right)$, which is

The prediction of this model-sampling the beam in steps of $10 \mathrm{~km}$-is presented in Fig. 10 (bottom). The associated MUSE observation was performed at an altitude of $82^{\circ}$, so the beam altitudes $h_{L}$ computed via our model are off by only $\cos \left(90^{\circ}-82^{\circ}\right) \equiv 1 \%$. The size of the out-of-focus LGS donut of 18 arc sec in diameter is accurately reproduced. For clarity, we sampled the primary mirror with only its center point and 60 locations on the outer edge of the mirror (see Fig. 11), giving rise to the specific ringlike patterns visible in Fig. 10. We note that to match the orientation of the beam in the observation, we rotated our final model by accounting for the value of the parallactic angle $\eta$ at the time of the observation. The Rayleigh (and Raman) beam is best matched by the model out to $30 \pm 5 \mathrm{~km}$ above ground (bottom-right panel). For higher altitudes, we do not 


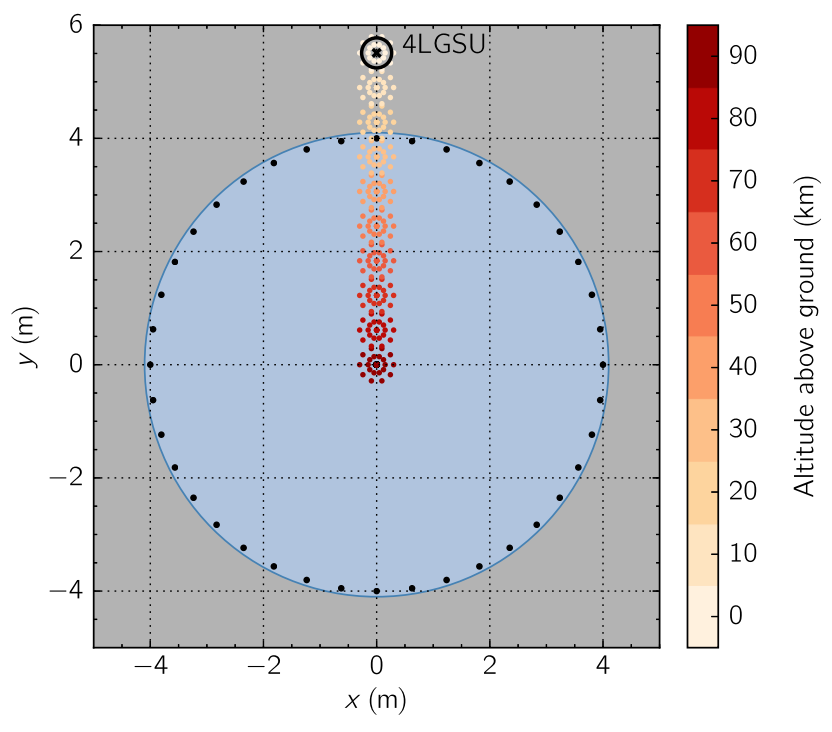

FIG. 11. Sampling of the primary mirror plane in our 4LGSF + UT4 model, resulting in the sampling of the reconstructed laser beam images shown in Fig. 10. For clarity, the mirror is only sampled using its center and 60 locations on its outer rim.

detect any evident sign of emission. For completeness, a movie illustrating the prediction of our model—sampling the full laser beam at $1-\mathrm{km}$ intervals-can be found in Ref. [95].

[1] M. C. Liu, Astronomical Science with Laser Guide Star Adaptive Optics: A Brief Review, a Current Snapshot, and a Bright Future, Proc. SPIE Int. Soc. Opt. Eng. 6272, 62720H (2006).

[2] M. C. Liu, LGS AO Science Impact: Present and Future Perspectives, Proc. SPIE Int. Soc. Opt. Eng. 7015, 701508 (2008).

[3] R. Foy and A. Labeyrie, Feasibility of Adaptive Telescope with Laser Probe, Astron. Astrophys. 152, L29 (1985).

[4] R. Davies and M. Kasper, Adaptive Optics for Astronomy, Annu. Rev. Astron. Astrophys. 50, 305 (2012).

[5] C. d'Orgeville and G. J. Fetzer, Four Generations of Sodium Guide Star Lasers for Adaptive Optics in Astronomy and Space Situational Awareness, Proc. SPIE Int. Soc. Opt. Eng. 9909, 99090R (2016).

[6] P. L. Wizinowich, D. Le Mignant, A. H. Bouchez, R. D. Campbell, J. C. Y. Chin, A. R. Contos, M. A. van Dam, S. K. Hartman, E. M. Johansson, R. E. Lafon et al., The W. M. Keck Observatory Laser Guide Star Adaptive Optics System: Overview, Publ. Astron. Soc. Pac. 118, 297 (2006).

[7] M. A. van Dam, A. H. Bouchez, D. Le Mignant, E. M. Johansson, P. L. Wizinowich, R. D. Campbell, J. C. Y. Chin, S. K. Hartman, R. E. Lafon, P. J. Stomski, Jr. et al., The W. M. Keck Observatory Laser Guide Star Adaptive Optics System: Performance Characterization, Publ. Astron. Soc. Pac. 118, 310 (2006).

[8] M. Boccas, F. Rigaut, M. Bec, B. Irarrazaval, E. James, A. Ebbers, C. d'Orgeville, K. Grace, G. Arriagada, S. Karewicz et al., Laser Guide Star Upgrade of Altair at Gemini North, Proc. SPIE Int. Soc. Opt. Eng. 6272, 62723L (2006).

[9] J. C. Christou, B. Neichel, F. Rigaut, M. Sheehan, R. M. McDermid, G. Trancho, C. Trujillo, and B. Walls, ALTAIR Performance and Updates at Gemini North, Proc. SPIE Int. Soc. Opt. Eng. 7736, 77361R (2010).

[10] C. d'Orgeville, S. Diggs, V. Fesquet, B. Neichel, W. Rambold, F. Rigaut, A. Serio, C. Araya, G. Arriagada, R. Balladares et al., Gemini South Multi-conjugate Adaptive Optics (GeMS) Laser Guide Star Facility On-Sky Performance Results, Proc. SPIE Int. Soc. Opt. Eng. 8447, 84471Q (2012).

[11] F. Rigaut, B. Neichel, M. Boccas, C. d'Orgeville, F. Vidal, M. A. van Dam, G. Arriagada, V. Fesquet, R. L. Galvez, G. Gausachs et al., Gemini Multiconjugate Adaptive Optics System Review-I. Design, Trade-offs and Integration, Mon. Not. R. Astron. Soc. 437, 2361 (2014).

[12] B. Neichel, F. Rigaut, F. Vidal, M. A. van Dam, V. Garrel, E. R. Carrasco, P. Pessev, C. Winge, M. Boccas, C. d'Orgeville et al., Gemini Multiconjugate Adaptive Optics System Review-II. Commissioning, Operation and Overall Performance, Mon. Not. R. Astron. Soc. 440, 1002 (2014).

[13] Y. Hayano, H. Takami, S. Oya, M. Hattori, Y. Saito, M. Watanabe, O. Guyon, Y. Minowa, S. E. Egner, M. Ito et al., Commissioning Status of Subaru Laser Guide Star Adaptive Optics System, Proc. SPIE Int. Soc. Opt. Eng. 7736, 77360N (2010).

[14] Y. Minowa, Y. Hayano, H. Terada, T.-S. Pyo, S. Oya, M. Hattori, M. Shirahata, H. Takami, O. Guyon, V. Garrel et al., Subaru Laser Guide Adaptive Optics System: Performance and Science Operation, Proc. SPIE Int. Soc. Opt. Eng. 8447, 84471F (2012).

[15] S. Lewis, D. B. Calia, B. Buzzoni, P. Duhoux, G. Fischer, I. Guidolin, A. Haimerl, W. Hackenberg, R. Hinterschuster, R. Holzlöhner et al., Laser Guide Star Facility Upgrade, The Messenger 155, 6 (2014).

[16] D. Bonaccini Calia, W. Hackenberg, R. Holzlöhner, S. Lewis, and T. Pfrommer, The Four-Laser Guide Star Facility: Design Considerations and System Implementation, Adv. Opt. Technol. 3, 345 (2014).

[17] Y. Feng, L. R. Taylor, and D. Bonaccini Calia, 25 W RamanFiber-Amplifier-Based $589 \mathrm{~nm}$ Laser for Laser Guide Star, Opt. Express 17, 19021 (2009).

[18] L. Taylor, Y. Feng, and D. Bonaccini Calia, High Power Narrowband 589 nm Frequency Doubled Fibre Laser Source, Opt. Express 17, 14687 (2009).

[19] D. Bonaccini Calia, Y. Feng, W. Hackenberg, R. Holzlöhner, L. Taylor, and S. Lewis, Laser Development for Sodium Laser Guide Stars at ESO, The Messenger 139, 12 (2010).

[20] R. Arsenault, P.-Y. Madec, J. Paufique, P. La Penna, S. Stroebele, E. Vernet, J.-F. Pirard, W. Hackenberg, H. Kuntschner, J. Kolb et al., The ESO Adaptive Optics Facility under Test, in Proceedings of the Third AO4ELT Conference (2013), p. 118, http://adsabs.harvard.edu/abs/ 2013aoel.confE.118A.

[21] R. Arsenault, R. Biasi, D. Gallieni, A. Riccardi, P. Lazzarini, N. Hubin, E. Fedrigo, R. Donaldson, S. Oberti, S. Stroebele et al., A Deformable Secondary Mirror for the VLT, Proc. SPIE Int. Soc. Opt. Eng. 6272, 62720V (2006). 
[22] R. Briguglio, R. Biasi, M. Xompero, A. Riccardi, M. Andrighettoni, D. Pescoller, G. Angerer, D. Gallieni, E. Vernet, J. Kolb et al., The Deformable Secondary Mirror of VLT: Final Electro-Mechanical and Optical Acceptance Test Results, Proc. SPIE Int. Soc. Opt. Eng. 9148, 914845 (2014).

[23] R. Stuik, R. Bacon, R. Conzelmann, B. Delabre, E. Fedrigo, N. Hubin, M. Le Louarn, and S. Ströbele, GALACSI - The Ground Layer Adaptive Optics System for MUSE, New Astron. Rev. 49, 618 (2006).

[24] P. La Penna, E. Aller Carpentier, J. Argomedo, R. Arsenault, R. D. Conzelmann, B. Delabre, R. Donaldson, F. Gago, P. Gutierrez-Cheetam, N. Hubin et al., AOF: Standalone Test Results of GALACSI, Proc. SPIE Int. Soc. Opt. Eng. 9909, 99092Z (2016).

[25] J. Paufique, A. Bruton, A. Glindemann, A. Jost, J. Kolb, L. Jochum, M. Le Louarn, M. Kiekebusch, N. Hubin, P.-Y. Madec et al., GRAAL: A Seeing Enhancer for the NIR WideField Imager Hawk-I, Proc. SPIE Int. Soc. Opt. Eng. 7736, 77361P (2010).

[26] J. Paufique, P.-Y. Madec, J. Kolb, H. Kuntschner, J. Argomedo, M. J. Kiekebusch, R. H. Donaldson, R. Arsenault, R. Siebenmorgen, C. Soenke et al., GRAAL on the Mountaintop, Proc. SPIE Int. Soc. Opt. Eng. 9909, 99092H (2016).

[27] P. Hibon, J. Paufique, H. Kuntschner, D. Dobrzycka, M. Le Louarn, E. Valenti, M. Neeser, E. Pompei, R. Arsenault, R. Siebenmorgen et al., Performance Updates of HAWK-I and Preparation for the Commissioning of the System GRAAL + HAWK-I, Proc. SPIE Int. Soc. Opt. Eng. 9908, 99080F (2016).

[28] R. Bacon, M. Accardo, L. Adjali, H. Anwand, S. Bauer, I. Biswas, J. Blaizot, D. Boudon, S. Brau-Nogue, J. Brinchmann et al., The MUSE Second-Generation VLT Instrument, Proc. SPIE Int. Soc. Opt. Eng. 7735, 773508 (2010).

[29] M. Kissler-Patig, J.-F. Pirard, M. Casali, A. Moorwood, N. Ageorges, C. Alves de Oliveira, P. Baksai, L. R. Bedin, E. Bendek, P. Biereichel et al., HAWK-I: The High-Acuity Wide-Field K-Band Imager for the ESO Very Large Telescope, Astron. Astrophys. 491, 941 (2008).

[30] R. Siebenmorgen, G. Carraro, E. Valenti, M. Petr-Gotzens, G. Brammer, E. Garcia, and M. Casali, The Science Impact of HAWK-I, The Messenger 144, 9 (2011).

[31] R. Arsenault, N. Hubin, S. Stroebele, E. Fedrigo, S. Oberti, M. Kissler-Patig, R. Bacon, R. McDermid, D. BonacciniCalia, R. Biasi et al., The VLT Adaptive Optics Facility Project: Adaptive Optics Modules, The Messenger 123, 11 (2006).

[32] F. Eisenhauer, R. Abuter, K. Bickert, F. Biancat-Marchet, H. Bonnet, J. Brynnel, R. D. Conzelmann, B. Delabre, R. Donaldson, J. Farinato et al., SINFONI-Integral Field Spectroscopy at 50 Milli-arcsecond Resolution with the ESO VLT, in Instrument Design and Performance for Optical/Infrared Ground-Based Telescopes, Proc. SPIE Int. Soc. Opt. Eng. 4841, 1548 (2003).

[33] H. Bonnet, R. Abuter, A. Baker, W. Bornemann, A. Brown, R. Castillo, R. Conzelmann, R. Damster, R. Davies, B. Delabre et al., First Light of SINFONI at the VLT, The Messenger 117, 17 (2004).
[34] Y. Hayano, W. Gaessler, H. Takami, N. Takato, and Y. Minowa, Rayleigh Scatter Measurement of Keck LGS by Subaru Telescope, Proc. SPIE Int. Soc. Opt. Eng. 4839, 452 (2003).

[35] D. M. Coulson and K. C. Roth, Gemini North R-Band Imaging of the Keck II Laser, in Adaptive Optics Systems II, Proc. SPIE Int. Soc. Opt. Eng. 7736, 773652 (2010).

[36] P. Amico, P. Santos, D. Summers, P. Duhoux, R. Arsenault, T. Bierwirth, H. Kuntschner, P.-Y. Madec, M. Prümm, and M. Rejkuba, The First Component of the Adaptive Optics Facility Enters Operations: The Laser Traffic Control System on Paranal, The Messenger 162, 19 (2015).

[37] R. Holzlöhner, S. M. Rochester, D. Bonaccini Calia, D. Budker, J. M. Higbie, and W. Hackenberg, Optimization of CW Sodium Laser Guide Star Efficiency, Astron. Astrophys. 510, A20 (2010).

[38] K. P. Birch and M. J. Downs, Letter to the Editor: Correction to the Updated Edlén Equation for the Refractive Index of Air, Metrologia 31, 315 (1994).

[39] D. A. Leonard, Observation of Raman Scattering from the Atmosphere Using a Pulsed Nitrogen Ultraviolet Laser, Nature (London) 216, 142 (1967).

[40] J. Cooney, Measurements on the Raman Component of Laser Atmospheric Backscatter, Appl. Phys. Lett. 12, 40 (1968).

[41] S. H. Melfi, J. Lawrence, and M. McCormick, Observation of Raman Scattering by Water Vapor in the Atmosphere, Appl. Phys. Lett. 15, 295 (1969).

[42] R. T. H. Collis, Lidar, Appl. Opt. 9, 1782 (1970).

[43] S. H. Melfi, Remote Measurements of the Atmosphere Using Raman Scattering, Appl. Opt. 11, 1605 (1972).

[44] A. M. Farah, D. D. Venable, A. N. Thorpe, F. Marsh, and W. S. Heaps, Validation of a Novel Ultraviolet Lidar System with Relative Raman-Scattering Cross Sections Determined from Atmospheric Measurements, Appl. Opt. 41, 407 (2002).

[45] D. Turner and D. Whiteman, Remote Raman Spectroscopy: Profiling Water Vapor and Aerosols in the Troposphere Using Raman Lidars, in Handbook of Vibrational Spectroscopy, edited by J. M. Chalmers and P. R. Griffiths (John Wiley \& Sons, Chichester, 2006).

[46] F. Rasetti, Incoherent Scattered Radiation in Diatomic Molecules, Phys. Rev. 34, 367 (1929).

[47] G. Herzberg, Molecular Spectra and Molecular Structure. Vol. 1: Spectra of Diatomic Molecules, 2nd ed. (Van Nostrand Reinhold, New York, 1950).

[48] D. N. Whiteman, S. H. Melfi, and R. A. Ferrare, Raman Lidar System for the Measurement of Water Vapor and Aerosols in the Earth's Atmosphere, Appl. Opt. 31, 3068 (1992).

[49] P. Keckhut, M. L. Chanin, and A. Hauchecorne, Stratosphere Temperature Measurement Using Raman Lidar, Appl. Opt. 29, 5182 (1990).

[50] F. Liu and F. Yi, Lidar-Measured Atmospheric $\mathrm{N}_{2}$ Vibrational-Rotational Raman Spectra and Consequent Temperature Retrieval, Opt. Express 22, 27833 (2014).

[51] See, for example, The International Standard Atmosphere Model, ISO 2533:1975. 
[52] K.-P. Huber and G. Herzberg, Molecular Spectra and Molecular Structure. Vol. 4: Constants of Diatomic Molecules (Van Nostrand Reinhold, New York, 1979).

[53] K. K. Irikura, Experimental Vibrational Zero-Point Energies: Diatomic Molecules, J. Phys. Chem. Ref. Data 36, 389 (2007).

[54] J. J. Barrett and N. I. Adams, Laser-Excited RotationVibration Raman Scattering in Ultra-Small Gas Samples, J. Opt. Soc. Am. 58, 311 (1968).

[55] D. Bonaccini Calia, M. Centrone, F. Pedichini, A. Ricciardi, A. Cerruto, and F. Ambrosino, Laser Guide Star Pointing Camera for ESO LGS Facilities, Proc. SPIE Int. Soc. Opt. Eng. 9148, 91483P (2014).

[56] M. Centrone, D. Bonaccini Calia, F. Pedichini, A. Cerruto, A. Ricciardi, and F. Ambrosino, Laser Pointing Camera: A Valuable Tool for the LGS-AO Operations, Proc. SPIE Int. Soc. Opt. Eng. 9909, 99095L (2016).

[57] D. Creek and R. Nicholls, A Comprehensive Re-analysis of the $O 2$ (...) Schumann-Runge Band System, Proc. R. Soc. A 341, 517 (1975).

[58] W. Knippers, K. van Helvoort, and S. Stolte, Vibrational Overtones of the Homonuclear Diatomics $\left(\mathrm{N}_{2}, \mathrm{O}_{2}, \mathrm{D}_{2}\right)$ Observed by the Spontaneous Raman Effect, Chem. Phys. Lett. 121, 279 (1985).

[59] W. S. Heaps and J. Burris, Airborne Raman Lidar, Appl. Opt. 35, 7128 (1996).

[60] G. Herzberg, Molecular Spectra and Molecular Structure. Vol. 2: Infrared and Raman Spectra of Polyatomic Molecules (Van Nostrand Reinhold, New York, 1945).

[61] C. M. Penney and M. Lapp, Raman-Scattering Cross Sections for Water Vapor, J. Opt. Soc. Am. 66, 422 (1976).

[62] I. Appenzeller, K. Fricke, W. Fürtig, W. Gässler, R. Häfner, R. Harke, H.-J. Hess, W. Hummel, P. Jürgens, R.-P. Kudritzki et al., Successful Commissioning of FORS1The First Optical Instrument on the VLT, The Messenger 94, 1 (1998).

[63] W. Freudling, M. Romaniello, D. M. Bramich, P. Ballester, V. Forchi, C. E. García-Dabló, S. Moehler, and M. J. Neeser, Automated Data Reduction Workflows for Astronomy. The ESO Reflex Environment, Astron. Astrophys. 559, A96 (2013).

[64] J. Cooney, Measurement of Atmospheric Temperature Profiles by Raman Backscatter, J. Appl. Meteorol. 11, 108 (1972).

[65] K. Wei, M. Li, S.-Q. Chen, Y. Bo, F. Chen, J.-W. Zuo, Q. Bian, J. Yao, L.-C. Zhou, L. Wei et al., First Light for the Sodium Laser Guide Star Adaptive Optics System on the Lijiang 1.8m Telescope, Res. Astron. Astrophys. 16, 183 (2016).

[66] D. Gavel, R. Kupke, D. Dillon, A. Norton, C. Ratliff, J. Cabak, A. Phillips, C. Rockosi, R. McGurk, S. Srinath et al., ShaneAO: Wide Science Spectrum Adaptive Optics System for the Lick Observatory, in Adaptive Optics Systems IV, Proc. SPIE Int. Soc. Opt. Eng. 9148, 914805 (2014).

[67] D. T. Gavel, R. Kupke, A. R. Rudy, S. Srinath, D. Dillon, and L. A. Poyneer, Lick Observatory's Shane Telescope Adaptive Optics System (ShaneAO): Research Directions and Progress, Proc. SPIE Int. Soc. Opt. Eng. 9909, 99092W (2016).
[68] C. Boyer, S. Adkins, D. R. Andersen, J. Atwood, Y. Bo, P. Byrnes, K. Caputa, J. Cavaco, B. Ellerbroek, L. Gilles et al., Adaptive Optics Program at TMT, inAdaptive Optics Systems IV, Proc. SPIE Int. Soc. Opt. Eng. 9148, 91480X (2014).

[69] C. Boyer and B. Ellerbroek, Adaptive Optics Program Update at TMT, Proc. SPIE Int. Soc. Opt. Eng. 9909, 990908 (2016).

[70] A. H. Bouchez, D. S. Acton, R. Biasi, R. Conan, B. Espeland, S. Esposito, J. Filgueira, D. Gallieni, B. A. McLeod, E. Pinna et al., The Giant Magellan Telescope Adaptive Optics Program, in Adaptive Optics Systems IV, Proc. SPIE Int. Soc. Opt. Eng. 9148, 91480W (2014).

[71] E. Diolaiti, MAORY: A Multi-conjugate Adaptive Optics RelaY for the E-ELT, The Messenger 140, 28 (2010).

[72] N. A. Thatte, F. Clarke, I. Bryson, H. Schnetler, M. Tecza, R. M. Bacon, A. Remillieux, E. Mediavilla, J. M. Herreros Linares, S. Arribas et al., HARMONI: The First Light Integral Field Spectrograph for the E-ELT, Proc. SPIE Int. Soc. Opt. Eng. 9147, 914725 (2014).

[73] R. Davies, J. Schubert, M. Hartl, J. Alves, Y. Clénet, F. Lang-Bardl, H. Nicklas, J.-U. Pott, R. Ragazzoni, E. Tolstoy et al., MICADO: First Light Imager for the E-ELT, Proc. SPIE Int. Soc. Opt. Eng. 9908, $99081 Z$ (2016).

[74] B. Neichel, T. Fusco, J.-F. Sauvage, C. Correia, K. Dohlen, K. El-Hadi, L. Blanco, N. Schwartz, F. Clarke, N. A. Thatte et al., The Adaptive Optics Modes for HARMONI: From Classical to Laser Assisted Tomographic AO, Proc. SPIE Int. Soc. Opt. Eng. 9909, 990909 (2016).

[75] S. Rabien, N. Ageorges, L. Barl, U. Beckmann, T. Blümchen, M. Bonaglia, J. L. Borelli, J. Brynnel, L. Busoni, L. Carbonaro et al., ARGOS: The Laser Guide Star System for the LBT, Proc. SPIE Int. Soc. Opt. Eng. 7736, 77360E (2010).

[76] G. Orban de Xivry, S. Rabien, L. Busoni, W. Gaessler, M. Bonaglia, J. Borelli, M. Deysenroth, S. Esposito, H. Gemperlein, M. Kulas et al., First On-Sky Results with ARGOS at LBT, Proc. SPIE Int. Soc. Opt. Eng. 9909, 990936 (2016).

[77] C. Benn, D. Abrams, T. Agocs, D. Cano, T. Gregory, J. C. Guerra, O. Martin, T. Morris, R. Myers, S. Rix et al., GLAS/ NAOMI: Ground-Layer AO at the William Herschel Telescope, Proc. SPIE Int. Soc. Opt. Eng. 7015, 701523 (2008).

[78] C. Baranec, R. G. Dekany, R. S. Burruss, B. P. Bowler, M. van Dam, R. Riddle, J. C. Shelton, T. Truong, J. Roberts, J. Milburn et al., PULSE: The Palomar Ultraviolet Laser for the Study of Exoplanets, Proc. SPIE Int. Soc. Opt. Eng. 9148, 91481D (2014).

[79] A. Tokovinin, R. Tighe, P. Schurter, R. Cantarutti, N. van der Bliek, M. Martinez, E. Mondaca, A. Montané, and W. Naudy Cortés, SAM Sees the Light, Proc. SPIE Int. Soc. Opt. Eng. 7736, 77363L (2010).

[80] M. Arnaboldi, M. Capaccioli, D. Mancini, P. Rafanelli, R. Scaramella, G. Sedmak, and G. P. Vettolani, VST: VLT Survey Telescope, The Messenger 93, 30 (1998).

[81] K. Kuijken, OmegaCAM: ESO's Newest Imager, The Messenger 146, 8 (2011).

[82] P. Amico, R. D. Campbell, and J. C. Christou, Laser Operations at the 8-10m Class Telescopes Gemini, Keck, 
and the VLT: Lessons Learned, Old and New Challenges, Proc. SPIE Int. Soc. Opt. Eng. 7737, 77370A (2010).

[83] D. Summers, B. Gregory, P. J. Stomski, Jr., A. Brighton, R. J. Wainscoat, P. L. Wizinowich, W. Gaessler, J. Sebag, C. Boyer, T. Vermeulen et al., Implementation of a Laser Traffic Control System Supporting Laser Guide Star Adaptive Optics on Mauna Kea, Proc. SPIE Int. Soc. Opt. Eng. 4839, 440 (2003).

[84] D. Summers, N. Apostolakos, R. Rutten, and G. Talbot, Second Generation Laser Traffic Control: Algorithm Changes Supporting Mauna Kea, La Palma, and Future Multi-telescope Laser Sites, Proc. SPIE Int. Soc. Opt. Eng. 6272, 627244 (2006).

[85] T. Fusco, S. Meimon, Y. Clenet, M. Cohen, H. Schnetler, J. Paufique, V. Michau, J.-P. Amans, D. Gratadour, C. Petit et al., ATLAS: The E-ELT Laser Tomographic Adaptive Optics System, Proc. SPIE Int. Soc. Opt. Eng. 7736, 77360D (2010).

[86] S. Zieleniewski, N. Thatte, S. Kendrew, R. C. W. Houghton, A. M. Swinbank, M. Tecza, F. Clarke, and T. Fusco, HSIM: A Simulation Pipeline for the HARMONI Integral Field Spectrograph on the European ELT, Mon. Not. R. Astron. Soc. 453, 3754 (2015).

[87] L. Pasquini, G. Avila, A. Blecha, C. Cacciari, V. Cayatte, M. Colless, F. Damiani, R. de Propris, H. Dekker, P. di Marcantonio et al., Installation and Commissioning of FLAMES, the VLT Multifibre Facility, The Messenger 110, 1 (2002).

[88] D. Bonaccini Calia, I. Guidolin, A. Friedenauer, M. Hager, V. Karpov, T. Pfrommer, R. Holzlöhner, S. Lewis, W. Hackenberg, G. Lombardi et al., The ESO Transportable
LGS Unit for Measurements of the LGS Photon Return and Other Experiments, inModern Technologies in Space- and Ground-Based Telescopes and Instrumentation II, Proc. SPIE Int. Soc. Opt. Eng. 8450, 84501R (2012).

[89] J. D. Hunter, Matplotlib: A 2D Graphics Environment, Comput. Sci. Eng. 9, 90 (2007).

[90] Astropy Collaboration, T. P. Robitaille, E. J. Tollerud, P. Greenfield, M. Droettboom, E. Bray, T. Aldcroft, M. Davis, A. Ginsburg, A. M. Price-Whelan et al. (Astropy Collaboration), Astropy: A Community Python Package for Astronomy, Astron. Astrophys. 558, A33 (2013).

[91] T. Robitaille and E. Bressert, APLpy: Astronomical Plotting Library in Python, Astrophysics Source Code Library, ascl:1208.017 (2012).

[92] J. J. Moré, The Levenberg-Marquardt Algorithm: Implementation and Theory, in Numerical Analysis: Proceedings of the Biennial Conference Held at Dundee, 1977 (Springer, Berlin, Heidelberg, 1978), pp. 105-116.

[93] F. Bonnarel, P. Fernique, O. Bienaymé, D. Egret, F. Genova, M. Louys, F. Ochsenbein, M. Wenger, and J. G. Bartlett, The ALADIN Interactive Sky Atlas. A Reference Tool for Identification of Astronomical Sources, Astron. Astrophys. Suppl. Ser. 143, 33 (2000).

[94] W. A. Joye and E. Mandel, New Features of SAOImage DS9, in Astronomical Data Analysis Software and Systems XII, ASP. Conf. Ser. 295, 489 (2003).

[95] See Supplemental Material at http://link.aps.org/ supplemental/10.1103/PhysRevX.7.021044 for a movie of the modeled beam footprint sampling the altitude in steps of $1 \mathrm{~km}$. 